\title{
Access to Both Anomers of Pectenotoxin Spiroketals by Kinetic Spiroketalization
}

\author{
Petri M. Pihko* and Jatta E. Aho \\ Laboratory of Organic Chemistry, Helsinki University of Technology, P.O.B. 6100, FI-02015 HUT, \\ Finland \\ Petri.Pihko@hut.fi
}

\section{Supporting Information}

General Methods. All reactions were carried out under an argon atmosphere in flame-dried glassware, unless otherwise noted. Nonaqueous reagents were transferred under argon via syringe or cannula and dried prior to use. Toluene and $\mathrm{Et}_{3} \mathrm{~N}$ were distilled from Na. THF and $\mathrm{Et}_{2} \mathrm{O}$ were distilled from $\mathrm{Na}$ /benzophenone. $\mathrm{CH}_{2} \mathrm{Cl}_{2}$ and DMSO were distilled from $\mathrm{CaH}_{2}$. DMF was distilled from molecular sieves (4 $\AA$ ) and oxalyl chloride was fractionally distilled. A stock sample of dry TBHP was dried by the procedure of Sharpless and co-workers. ${ }^{1}$ All batches of TBHP were also dried on $4 \AA$ molecular sieves immediately prior to use. All of the self-made reagents were azeotropically dried with toluene. Other solvents and reagents were used as obtained from supplier, unless otherwise noted. Analytical TLC was performed using Merck silica gel F254 (230-400 mesh) plates and analyzed by UV light or by staining upon heating with vanillin solution $\left(6 \mathrm{~g}\right.$ vanillin, $5 \mathrm{~mL}$ conc. $\mathrm{H}_{2} \mathrm{SO}_{4}, 3 \mathrm{~mL}$ glacial acetic acid, $250 \mathrm{~mL}$ $\mathrm{EtOH})$ or with ninhydrin solution ( $1 \mathrm{~g}$ ninhydrin, $100 \mathrm{~mL}$ isopropanol, 5 drops glacial acetic acid). For silica gel chromatography, the flash chromatography technique was used, with Merck silica gel 60 (230400 mesh) and p.a. grade solvents unless otherwise noted.

The ${ }^{1} \mathrm{H}$ NMR and ${ }^{13} \mathrm{C}$ NMR spectra were recorded in either $\mathrm{CDCl}_{3}$ or $\mathrm{CD}_{3} \mathrm{CN}$ on a Bruker Avance 400 $\left({ }^{1} \mathrm{H} 399.98 \mathrm{MHz} ;{ }^{13} \mathrm{C} 100.59 \mathrm{MHz}\right)$ spectrometer. The chemical shifts are reported in ppm relative to $\mathrm{CHCl}_{3}\left(\delta\right.$ 7.26) or $\mathrm{CHD}_{2} \mathrm{CN}\left(\delta\right.$ 1.94) for ${ }^{1} \mathrm{H}$ NMR. For the ${ }^{13} \mathrm{C}$ NMR spectra, the residual $\mathrm{CDCl}_{3}(\delta 77.0)$ or $\mathrm{CD}_{3} \mathrm{CN}(\delta$ 118.26) were used as the internal standard. The enantiomeric excess (ee) of the products were determined by HPLC in comparison to the corresponding racemic samples using Waters 501 pump and Waters 486 detector, Daicel Chiralcel AD or OD columns and $i-\mathrm{PrOH} /$ hexane as eluent. IR spectra were recorded on a Perkin-Elmer Spectrum One spectrometer. Optical rotations were obtained with a Perkin-Elmer 343 polarimeter. High resolution mass spectrometric data were obtained by the University of Oulu on Micromass LCT spectrometer. The elemental analyses were performed at the Analytical Services of the Department of Chemical Technology, Laboratory of Organic Chemistry.

The racemic samples corresponding to the compounds 18, 19, and 20 were prepared using $m$-CPBA (120 mol\% in $\mathrm{CH}_{2} \mathrm{Cl}_{2}$ ) as the oxidant for 17. All racemic samples were purified and all subsequent reactions were performed in a manner identical to their enantioenriched counterparts. 


\section{5-Benzyloxypentanol}

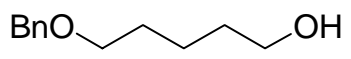

To neat 1,5-pentanediol $(47.3 \mathrm{~mL}, 46.9 \mathrm{~g}, 450 \mathrm{mmol})$ at $\mathrm{rt}$ was added both benzyl bromide $(15.3 \mathrm{~mL}$, $22.1 \mathrm{~g}, 129 \mathrm{mmol})$ and powdered $\mathrm{KOH}(30.4 \mathrm{~g}, 542 \mathrm{mmol})$ in four equal portions over $1 \mathrm{~h}$. After the last addition the solution was stirred for an additional $4 \mathrm{~h} . \mathrm{H}_{2} \mathrm{O}(75 \mathrm{~mL})$ was then added. The separated aqueous phase was extracted with EtOAc $(4 \times 30 \mathrm{~mL})$ and the combined organic phases were dried over $\mathrm{MgSO}_{4}$, filtered and concentrated. Silica gel column chromatography (30-70\% ethyl acetate in hexanes) yielded 5-benzyloxypentanol $(20.1 \mathrm{~g}, 80 \%)$ as a pale yellow oil. IR- and ${ }^{1} \mathrm{H}$ NMR-data match those reported in literature. ${ }^{2}$

\section{5-(Benzyloxy)pentanal 15}

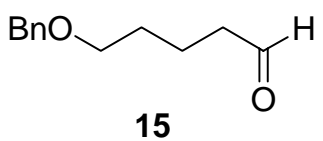

A solution of oxalyl chloride $(1.9 \mathrm{~mL}, 2.74 \mathrm{~g}, 21.6 \mathrm{mmol})$ in $\mathrm{CH}_{2} \mathrm{Cl}_{2}(20 \mathrm{~mL})$ was cooled to $-55^{\circ} \mathrm{C}$ and DMSO (3.1 mL, 3.37mg, $43.1 \mathrm{mmol})$ was added. After $5 \mathrm{~min}$, a solution of 5-benzyloxypentanol (3.81 g, $19.6 \mathrm{mmol})$ in $\mathrm{CH}_{2} \mathrm{Cl}_{2}(45 \mathrm{~mL})$ was added. The resulting mixture was stirred for $15 \mathrm{~min}$. Triethylamine $(13.1 \mathrm{~mL}, 9.52 \mathrm{~g}, 94.1 \mathrm{mmol})$ was then added dropwise. Stirring was continued at $-55^{\circ} \mathrm{C}$ for an additional $10 \mathrm{~min}$ and then the mixture was allowed to warm to $\mathrm{rt} . \mathrm{H}_{2} \mathrm{O}(100 \mathrm{~mL})$ was added and the separated aqueous phase was extracted with $\mathrm{CH}_{2} \mathrm{Cl}_{2}(2 \times 60 \mathrm{~mL})$. The combined organic phases were washed with brine $(2 \times 60 \mathrm{~mL})$, dried over $\mathrm{MgSO}_{4}$, filtered and concentrated. Silica gel column chromatography $(30 \%$ ethyl acetate in hexanes) yielded 5-(benzyloxy)pentanal $15(3.5 \mathrm{~g}, 93 \%)$ as a pale yellow oil. IR- and ${ }^{1} \mathrm{H}$ NMR-data match those reported in the literature. ${ }^{2}$

\section{7-(Benzyloxy)-methyl-2-(Z)-hepteonate 16}

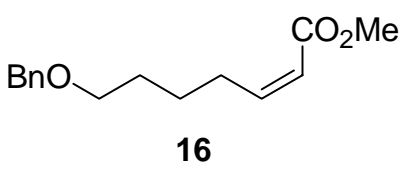

To a solution of bis(o-cresyl) phosphonoacetate $(7.4 \mathrm{~mL}, 9.03 \mathrm{~g}, 27.0 \mathrm{mmol})$ in THF $(150 \mathrm{~mL})$ at $0{ }^{\circ} \mathrm{C}$ was added $\mathrm{NaI}(3.12 \mathrm{~g}, 20.8 \mathrm{mmol})$. After $5 \mathrm{~min}, \mathrm{NaH}(60 \%$ dispersion, $0.65 \mathrm{~g}, 27 \mathrm{mmol})$ was slowly added and the resulting mixture was cooled $-78^{\circ} \mathrm{C}$. A solution of 5-(benzyloxy)pentanal 15 (4.0 g, 20.8 $\mathrm{mmol})$ in THF $(15 \mathrm{~mL})$ was added dropwise via cannula. The resulting solution was stirred at $-78^{\circ} \mathrm{C}$ for an additional $8 \mathrm{~h}$ before half-saturated $\mathrm{NH}_{4} \mathrm{Cl}(120 \mathrm{~mL})$ was added. $\mathrm{H}_{2} \mathrm{O}(50 \mathrm{~mL})$ was added to get a clear solution and the mixture was allowed to warm to rt. The separated aqueous phase was extracted with $\mathrm{Et}_{2} \mathrm{O}$ ( $3 \times 80 \mathrm{~mL}$ ). The combined organic phases were dried over $\mathrm{Na}_{2} \mathrm{SO}_{4}$, filtered and concentrated. Silica gel column chromatography (5\% ethyl acetate in hexanes) yielded 7-(benzyloxy)-methyl-2-(Z)-heptenoate 16 (4.39 $\mathrm{g}, 85 \%, \mathrm{Z} / \mathrm{E} 97 / 3)$ as a pale yellow oil. 
$R_{f}=0.63$ (50\% EtOAc in hexanes, UV/vanillin); IR (thin film, $\mathrm{cm}^{-1}$ ) 2944, 2859, 1723, 1645, 1438, 1199, 1170, 1103, 736, 698; ${ }^{1} \mathrm{H}$ NMR (400 MHz, $\left.\mathrm{CDCl}_{3}\right) \delta$ 7.37-7.25 (m, 5H), $6.22(\mathrm{dt}, 1 \mathrm{H}, J=11.5 \mathrm{~Hz}$, $7.5 \mathrm{~Hz}), 5.78$ (dt, 1H, J=11.5 Hz, $1.7 \mathrm{~Hz}), 4.50$ (s, 2H), 3.70 (s, 3H), 3.49 (t, 2H, J=6.4 Hz), 2.68 (dq, $2 \mathrm{H}, J=1.7 \mathrm{~Hz}, 7.5 \mathrm{~Hz}), 1.70-1.63(\mathrm{~m}, 2 \mathrm{H}), 1.60-1.50(\mathrm{~m}, 2 \mathrm{H}) ;{ }^{13} \mathrm{C} \mathrm{NMR}\left(100 \mathrm{MHz}, \mathrm{CDCl}_{3}\right) \delta 166.8$, $150.4,138.6,128.3,127.6,127.5,119.4,72.9,70.0,51.0,29.3,28.7,25.6$; HRMS (ESI) calcd. for $\mathrm{C}_{15} \mathrm{H}_{20} \mathrm{O}_{3} \mathrm{Na} 271.1310$, found 271.1307, $\Delta=1.1 \mathrm{ppm}$.

\section{7-(Benzyloxy)-methyl-2-(Z)-hepten-1-ol 17}

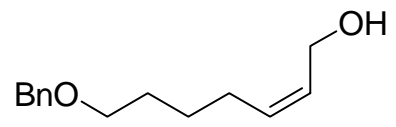

17

A solution of enoate $16(2.86 \mathrm{~g}, 11.5 \mathrm{mmol})$ in THF $(150 \mathrm{~mL})$ was cooled to $-78{ }^{\circ} \mathrm{C}$ and DIBAL-H (28.8 $\mathrm{mL}$ of $1 \mathrm{M}$ solution in toluene, $28.8 \mathrm{mmol}$ ) was added dropwise over a period of $10 \mathrm{~min}$. The resulting solution was stirred at $-78^{\circ} \mathrm{C}$ for an additional $45 \mathrm{~min}$ and then warmed to $0{ }^{\circ} \mathrm{C}$. After $1.5 \mathrm{~h}$, the reaction was quenched with aqueous saturated Rochelle's salt $(100 \mathrm{~mL})$. The mixture was warmed to $\mathrm{rt}$ and stirred for an additional $1.5 \mathrm{~h}$ before the solution was extracted with EtOAc $(3 \times 60 \mathrm{~mL})$. The combined organic phases were washed with $\mathrm{H}_{2} \mathrm{O}$, saturated aqueous $\mathrm{NaHCO}_{3}$ and brine $(60 \mathrm{~mL}$ each), dried over $\mathrm{MgSO}_{4}$, filtered and concentrated. Silica gel column chromatography (40\% MTBE in hexanes) yielded 7-(benzyloxy)-methyl-2-(Z)-hepten-1-ol 17 (2.39 g, 94\%) as a pale yellow oil.

$R_{f}=0.33$ (50\% EtOAc in hexanes, UV/vanillin); IR (thin film, $\mathrm{cm}^{-1}$ ) 3369, 2935, 2859, 1454, 1102, 1027, 735, 697; ${ }^{1} \mathrm{H}$ NMR (400 MHz, $\left.\mathrm{CDCl}_{3}\right) \delta$ 7.37-7.28 (m, 5H), 5.65-5.50 (m, 2H), 4.50 (s, 2H), 4.18 (d, 2H, $J=6.6 \mathrm{~Hz}), 3.47$ (t, 2H, $J=6.4 \mathrm{~Hz}), 2.10(\mathrm{q}, 2 \mathrm{H}, J=7.3 \mathrm{~Hz}), 1.66-1.59(\mathrm{~m}, 2 \mathrm{H}), 1.50-1.43$ (m, $3 \mathrm{H}) ;{ }^{13} \mathrm{C} \mathrm{NMR}\left(100 \mathrm{MHz}, \mathrm{CDCl}_{3}\right) \delta 138.6,132.8,128.7,128.3,127.6,127.5,72.9,70.2,58.5,29.2,27.2$, 26.2; HRMS (ESI) calcd. for $\mathrm{C}_{14} \mathrm{H}_{20} \mathrm{O}_{2} \mathrm{Na} 243.1361$, found 243.1360, $\Delta=0.4 \mathrm{ppm}$.

\section{Epoxide 18}

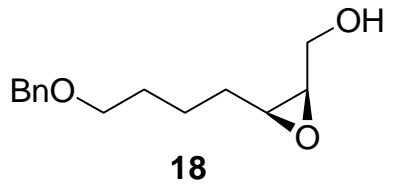

To a stirred $0{ }^{\circ} \mathrm{C}$ solution of crushed $4 \AA$ molecular sieves $(0.6 \mathrm{~g})$ in $\mathrm{CH}_{2} \mathrm{Cl}_{2}(20 \mathrm{~mL})$ were added D-(-)diethyltartrate $(0.23 \mathrm{~mL}, 0.28 \mathrm{~g}, 1.36 \mathrm{mmol})$ and $\mathrm{Ti}(\mathrm{OiPr})_{4}(0.27 \mathrm{~mL}, 0.26 \mathrm{~g}, 0.91 \mathrm{mmol})$. This mixture was cooled to $-20{ }^{\circ} \mathrm{C}$ and tert-butyl hydroperoxide $(1.8 \mathrm{~mL}$ of a $\sim 5.0 \mathrm{M}$ solution in isooctane, $\sim 9.08 \mathrm{mmol}$ ) was added. The resulting mixture was stirred for $20 \mathrm{~min}$ before a solution of allylic alcohol 17 (1.0 g, $4.54 \mathrm{mmol})$ in $\mathrm{CH}_{2} \mathrm{Cl}_{2}(5 \mathrm{~mL})$ was added dropwise via cannula. The allylic alcohol was first azeotropically dried with toluene and then dissolved in $\mathrm{CH}_{2} \mathrm{Cl}_{2}$ and dried over $4 \AA$ molecular sieves. The resulting mixture was stirred at $-20{ }^{\circ} \mathrm{C}$ for an additional $70 \mathrm{~h}$ and then warmed to $0{ }^{\circ} \mathrm{C} . \mathrm{H}_{2} \mathrm{O}(4 \mathrm{~mL})$ was 
added and the stirring was continued at $0{ }^{\circ} \mathrm{C}$ for an additional $1 \mathrm{~h}$. The mixture was allowed to warm to rt. A solution of $30 \% \mathrm{NaOH}$ in saturated aqueous $\mathrm{NaCl}(1 \mathrm{~mL})$ was added and the resulting mixture was stirred for $45 \mathrm{~min}$. The solution was diluted with $\mathrm{H}_{2} \mathrm{O}(4 \mathrm{~mL})$ and filtered to get a better separation of the two phases. The phases were separated and the aqueous phase was extracted with $\mathrm{CH}_{2} \mathrm{Cl}_{2}(3 \times 20 \mathrm{~mL})$. The combined organic phases were dried over $\mathrm{Na}_{2} \mathrm{SO}_{4}$, filtered and concentrated. Silica gel column chromatography ( $40 \%$ ethyl acetate in hexanes) yielded epoxide $18(0.67 \mathrm{~g}, 62 \%, 83 \%$ ee) as a colorless oil.

$R_{f}=0.27\left(70 \%\right.$ EtOAc in hexanes UV/vanillin); $[\alpha]_{\mathrm{D}}=+2.5\left(\right.$ c $\left.0.59, \mathrm{CH}_{2} \mathrm{Cl}_{2}\right) ; \mathrm{IR}$ (thin film, $\left.\mathrm{cm}^{-1}\right) 3436$, 2932, 2861, 1727, 1454, 1101, 1044, 737, 698; ${ }^{1} \mathrm{H}$ NMR (400 MHz, $\left.\mathrm{CDCl}_{3}\right) \delta$ 7.37-7.27 (m, 5H), 4.50 (s, 2H), 3.82 (ddd, 1H, J=4.5 Hz, 7.4 Hz, $12.0 \mathrm{~Hz}$ ), 3.68 (ddd, $1 \mathrm{H}, J=5.1 \mathrm{~Hz}, 6.8 \mathrm{~Hz}, 12.0 \mathrm{~Hz}$ ), 3.49 (t, 2H, $J=6.2 \mathrm{~Hz}), 3.14(\mathrm{dt}, 1 \mathrm{H}, J=4.3 \mathrm{~Hz}, 6.8 \mathrm{~Hz}), 3.03(\mathrm{~m}, 1 \mathrm{H}), 1.71-1.51(\mathrm{~m}, 6 \mathrm{H}) ;{ }^{13} \mathrm{C} \mathrm{NMR}(100 \mathrm{MHz}$, $\left.\mathrm{CDCl}_{3}\right) \delta 138.5,128.4,127.7,127.6,73.0,70.0,60.8,57.2,56.6,29.4,27.7,23.5$. These data match those

reported in literature. ${ }^{3}$ HRMS (ESI) calcd. for $\mathrm{C}_{15} \mathrm{H}_{20} \mathrm{O}_{3} \mathrm{Na} 259.1310$, found 259.1336, $\Delta=10 \mathrm{ppm}$. The enantiomeric purity was determined by HPLC (Daicel Chiralcel AD column, $15 \%$ i-PrOH/hexanes, flow rate $0.5 \mathrm{~mL} / \mathrm{min}$ ): $\tau_{\text {major }}=14.94 \mathrm{~min} ; \tau_{\operatorname{minor}}=16.85 \mathrm{~min}$.

\section{7-(Benzyloxy)-2-methyl-heptane-1,3-diol 19}

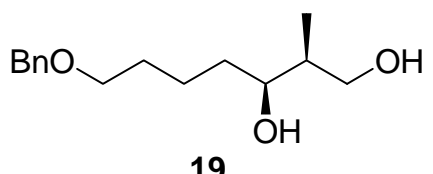

A mixture of $\mathrm{CuCN}(1.25 \mathrm{~g}, 14.0 \mathrm{mmol})$ in $\mathrm{Et}_{2} \mathrm{O}(35 \mathrm{~mL})$ was cooled to $-78{ }^{\circ} \mathrm{C}$ and methyllithium $\left(17.0 \mathrm{~mL}\right.$ of a $1.48 \mathrm{M}$ solution in $\left.\mathrm{Et}_{2} \mathrm{O}, 25.1 \mathrm{mmol}\right)$ was added dropwise. The resulting mixture was warmed to $-20{ }^{\circ} \mathrm{C}$ and stirred vigorously for $1 \mathrm{~h}$. The color of the solution turned pale green (some $\mathrm{CuCN}$ remained undissolved). A solution of epoxide $18(0.55 \mathrm{~g}, 2.33 \mathrm{mmol})$ in $\mathrm{Et}_{2} \mathrm{O}(15 \mathrm{~mL})$ was added via cannula and the stirring was continued at $-15^{\circ} \mathrm{C}$ for $2.5 \mathrm{~h}$. $\mathrm{Et}_{2} \mathrm{O}(15 \mathrm{~mL})$ and saturated aqueous $\mathrm{NH}_{4} \mathrm{Cl}(20$ $\mathrm{mL}$ ) were added. The resulting mixture was warmed to $\mathrm{rt}$ and filtered. The separated organic phase was washed with saturated aqueous $\mathrm{NH}_{4} \mathrm{Cl}, \mathrm{H}_{2} \mathrm{O}$ and brine $(2 \times 25 \mathrm{~mL}$ each). The combined aqueous phases were back-extracted with $\mathrm{Et}_{2} \mathrm{O}(25 \mathrm{~mL})$ and the combined organic phases were dried over $\mathrm{Na}_{2} \mathrm{SO}_{4}$, filtered and concentrated to yield the crude 19. To this crude material were added THF (15 mL), $\mathrm{H}_{2} \mathrm{O}(15$ $\mathrm{mL})$ and $\mathrm{NaIO}_{4}(0.16 \mathrm{~g}, 0.77 \mathrm{mmol})$ at $\mathrm{rt}$. The resulting solution was stirred for $1.5 \mathrm{~h}$ and then diluted with $\mathrm{Et}_{2} \mathrm{O}(20 \mathrm{~mL})$. The separated organic phase was washed with saturated aqueous $\mathrm{NaHCO}_{3}(25 \mathrm{~mL})$ and brine $(25 \mathrm{~mL})$. The combined aqueous phases were back-extracted with $\mathrm{Et}_{2} \mathrm{O}(25 \mathrm{~mL})$ and the combined organic phases were dried over $\mathrm{Na}_{2} \mathrm{SO}_{4}$, filtered and concentrated. Silica gel column chromatography $(50 \% \mathrm{MTBE}$ in hexanes), followed by recrystallization of the product from EtOAc/hexanes (1:10) yielded diol $19(0.38 \mathrm{~g}, 65 \%)$ as white crystalline solid. 
$R_{f}=0.23\left(70 \%\right.$ EtOAc in hexanes, UV/vanillin); mp 50-52 ${ }^{\circ} \mathrm{C} ;[\alpha]_{\mathrm{D}}=-5.7\left(\mathrm{c} 0.42, \mathrm{CH}_{2} \mathrm{Cl}_{2}\right)(95 \%$ ee $)$; IR (thin film, $\mathrm{cm}^{-1}$ ) 3370, 2937, 2864, 1455, 1101, 1028, 736, 698; ${ }^{1} \mathrm{H}$ NMR (400 MHz, $\mathrm{CDCl}_{3}$ ) $\delta 7.37-7.26$ $(\mathrm{m}, 5 \mathrm{H}), 4.50$ (s, 2H), $3.82(\mathrm{~m}, 1 \mathrm{H}), 3.69(\mathrm{~m}, 2 \mathrm{H}), 3.49$ (dt, 2H, $J=0.9 \mathrm{~Hz}, 12.7 \mathrm{~Hz}), 2.22(\mathrm{~s}, 2 \mathrm{H}), 1.82-$ $1.36(\mathrm{~m}, 7 \mathrm{H}), 0.9$ (d, 3H, $J=7.1 \mathrm{~Hz}) ;{ }^{13} \mathrm{C} \mathrm{NMR}\left(100 \mathrm{MHz}, \mathrm{CDCl}_{3}\right) \delta 138.5,128.4,127.7,127.5,74.5$, 72.9, 70.3, 67.2, 39.1, 33.8, 29.6, 22.9, 10.1; These data match those reported in literature ${ }^{3}$; HRMS (ESI) calcd. for $\mathrm{C}_{15} \mathrm{H}_{24} \mathrm{O}_{3} \mathrm{Na} 275.1623$, found 275.1628, $\Delta=1.8$ ppm; Anal. calcd. for $\mathrm{C}_{15} \mathrm{H}_{24} \mathrm{O}_{3}$ : C, 71.39; $\mathrm{H}$, 9.59; found: C, 71.62; H, 9.64.

\section{7-(Benzyloxy)-2-methyl-1-(tert-butyl-diphenylsilyl)-heptan-3-ol}<smiles>C[C@H](CO[PbH2])[C@@H](O)CCCCOc1ccccc1</smiles>

To a stirred $0{ }^{\circ} \mathrm{C}$ solution of diol $19(0.38 \mathrm{~g}, 1.51 \mathrm{mmol})$ in $\mathrm{CH}_{2} \mathrm{Cl}_{2}(6 \mathrm{~mL})$ were added triethylamine $(0.48 \mathrm{~mL}, 1.35 \mathrm{~g}, 3.46 \mathrm{mmol})$ and TBDPSCl $(0.47 \mathrm{~mL}, 0.5 \mathrm{~g}, 1.81 \mathrm{mmol})$. After additions the mixture was allowed to warm to rt and stirring was continued for an additional $14 \mathrm{~h}$. The mixture was diluted with $\mathrm{Et}_{2} \mathrm{O}(5 \mathrm{~mL})$ and washed with $\mathrm{H}_{2} \mathrm{O}(5 \mathrm{~mL})$. The aqueous phase was extracted with $\mathrm{Et}_{2} \mathrm{O}(5 \mathrm{~mL})$ and the combined organic phases were dried over $\mathrm{MgSO}_{4}$, filtered and concentrated. Silica gel column chromatography (30\% EtOAc in hexanes) yielded 7-(benzyloxy)-2-methyl-1-(tert-butyl-diphenylsilyl)heptan-3-ol (0.73 g, 98\%) as a pale yellow viscous oil.

$R_{f}=0.69$ (70 \% EtOAc in hexanes, UV/vanillin); $[\alpha]_{\mathrm{D}}=-3.4\left(\right.$ c $\left.0.44, \mathrm{CH}_{2} \mathrm{Cl}_{2}\right)$; IR (thin film, $\left.\mathrm{cm}^{-1}\right) 3460$, 2932, 2858, 1472, 1428, 1112, 740, 702; ${ }^{1} \mathrm{H}$ NMR (400 MHz, $\left.\mathrm{CDCl}_{3}\right) \delta$ 7.68-7.65 (m, 4H), 7.46-7.27 (m, $11 \mathrm{H}), 4.51(\mathrm{~s}, 2 \mathrm{H}), 3.86(\mathrm{~m}, 1 \mathrm{H}), 3.75(\mathrm{dd}, 1 \mathrm{H}, J=4.2 \mathrm{~Hz}, 10.1 \mathrm{~Hz}), 3.66(\mathrm{dd}, 1 \mathrm{H}, J=6.0 \mathrm{~Hz}, 10.1 \mathrm{~Hz})$, $3.49(\mathrm{dt}, 2 \mathrm{H}, J=1.0 \mathrm{~Hz}, 6.5 \mathrm{~Hz}), 2.77$ (d, 1H, $J=2.9 \mathrm{~Hz}), 1.79-1.36$ (m, 7H), $1.06(\mathrm{~s}, 9 \mathrm{H}), 0.90$ (d, 3H, $J$ $=7.2 \mathrm{~Hz}) ;{ }^{13} \mathrm{C} \mathrm{NMR}\left(100 \mathrm{MHz}, \mathrm{CDCl}_{3}\right) \delta 138.7,135.7,135.6,133.1,133.0,129.83,129.79,128.3$, 127.8, 127.6, 127.5, 74.1, 72.9, 70.4, 68.7, 39.1, 34.0, 29.8, 26.9, 22.9, 19.2, 10.2; HRMS (ESI) calcd. for $\mathrm{C}_{31} \mathrm{H}_{42} \mathrm{O}_{3} \mathrm{NaSi}$ 513.2801, found 513.2823, $\Delta=4.3 \mathrm{ppm}$.

\section{6-Methyl-7-(tert-butyl-diphenylsilyl)-heptane-1,5-diol 20}

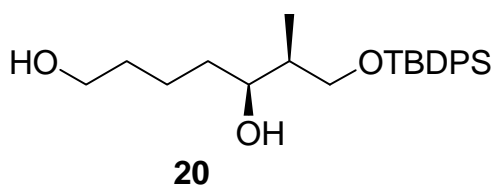

To a stirred solution of 7-(benzyloxy)-2-methyl-1-(tert-butyl-diphenylsilyl)-heptan-3-ol (0.66 g, $1.34 \mathrm{mmol})$ in EtOAc $(20 \mathrm{~mL})$ was added $\mathrm{Pd}(\mathrm{OH})_{2}$ on charcoal $(0.10 \mathrm{~g}$ of $20 \% \mathrm{Pd}$ catalyst, $0.15 \mathrm{mmol})$ under argon flow. The reaction flask was repeatedly evacuated and flushed with $\mathrm{H}_{2}$. The suspension was vigorously stirred under $\mathrm{H}_{2}$ atmosphere for $13 \mathrm{~h}$ and then filtered through Celite. The filter pad was washed with EtOAc ( 3 x $20 \mathrm{~mL})$ and the combined filtrates were concentrated. Silica gel column 
chromatography (40\% EtOAc in hexanes) yielded 6-methyl-7-(tert-butyl-diphenylsilyl)-heptane-1,5-diol $20(0.46 \mathrm{~g}, 89 \%)$ as a colorless viscous oil.

$R_{f}=0.33\left(70 \%\right.$ EtOAc in hexanes, UV/vanillin); $[\alpha]_{\mathrm{D}}=-3.4\left(\mathrm{c} 0.61, \mathrm{CH}_{2} \mathrm{Cl}_{2}\right)$; IR (thin film, $\left.\mathrm{cm}^{-1}\right) 3339$, 2931, 2585, 1428, 1112, 740, 701; ${ }^{1} \mathrm{H}$ NMR (400 MHz, $\left.\mathrm{CDCl}_{3}\right) \delta$ 7.68-7.65 (m, 4H), 7.46-7.38 (m, 6H), $3.87(\mathrm{~m}, 1 \mathrm{H}), 3.76(\mathrm{dd}, 1 \mathrm{H}, J=4.2 \mathrm{~Hz}, 10.1 \mathrm{~Hz}), 3.69-3.65(\mathrm{~m}, 3 \mathrm{H}), 2.02$ (br s, 2H), 1.80-1.71 (m, 1H), 1.66-1.35 (m, 6H), $1.06(\mathrm{~s}, 9 \mathrm{H}), 0.92(\mathrm{~d}, 3 \mathrm{H}, J=7.1 \mathrm{~Hz}) ;{ }^{13} \mathrm{C} \mathrm{NMR}\left(100 \mathrm{MHz}, \mathrm{CDCl}_{3}\right) \delta 135.7,135.6$, 133.1, 132.9, 129.9, 129.8, 127.8, 74.2, 68.7, 62.9, 39.2, 33.8, 32.7, 26.9, 22.5, 19.2, 10.3; HRMS (ESI) calcd. for $\mathrm{C}_{24} \mathrm{H}_{36} \mathrm{O}_{3} \mathrm{NaSi}$ 423.2331, found 423.2345, $\Delta=3.3 \mathrm{ppm}$. The enantiomeric purity was determined by HPLC (Daicel Chiralcel OD column, 1:9 $i-\mathrm{PrOH} /$ hexanes, flow rate $0.5 \mathrm{ml} / \mathrm{min}$ ): $\tau_{\text {major }}=$ $11.15 \mathrm{~min} ; \tau_{\text {minor }}=14.82 \mathrm{~min}, 95 \%$ ee.

\section{Lactone 21}

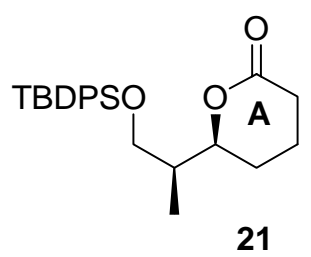

To a stirred solution of diol $20(0.70 \mathrm{~g}, 1.75 \mathrm{mmol})$ in $\mathrm{CH}_{2} \mathrm{Cl}_{2}(20 \mathrm{~mL})$ were added crushed $4 \AA$ molecular sieves $(0.75 \mathrm{~g})$ and pyridinium chlorochromate $(\mathrm{PCC}, 0.66 \mathrm{~g}, 3.06 \mathrm{mmol})$ at $\mathrm{rt}$. After $3 \mathrm{~h}$, second portions of molecular sieves $(0.25 \mathrm{~g})$ and PCC $(0.66 \mathrm{~g}, 3.06 \mathrm{mmol})$ were added. Stirring was continued for an additional $13 \mathrm{~h}$ and a third portion molecular sieves $(0.2 \mathrm{~g})$ and PCC $(0.57 \mathrm{~g}, 2.63 \mathrm{mmol})$ were added. After $3 \mathrm{~h}$, a third portion of PCC $(0.18 \mathrm{~g}, 1.88 \mathrm{mmol})$ was added. This mixture was stirred for an additional $3 \mathrm{~h}$ before the mixture was filtered through silica gel with EtOAc and then concentrated. Silica gel column chromatography (10\% EtOAc in hexanes) yielded lactone 21 (0.40 g, 58\%) as a colorless oil.

$R_{f}=0.56\left(70 \%\right.$ EtOAc in hexanes, UV/vanillin); $[\alpha]_{\mathrm{D}}=+26.3\left(\mathrm{c} \mathrm{1.15}, \mathrm{CH}_{2} \mathrm{Cl}_{2}\right) ; \mathrm{IR}$ (thin film, $\left.\mathrm{cm}^{-1}\right) 2958$, 2931, 2883, 2857, 1736, 1428, 1239, 1112, 702; ${ }^{1} \mathrm{H}$ NMR (400 MHz, $\left.\mathrm{CDCl}_{3}\right) \delta$ 7.67-7.63 (m, 4H), 7.457.37 (m, 6H), 4.49 (ddd, 1H, J=3.0 Hz, 4.1 Hz, 11.5 Hz), 3.73 (dd, 1H, J=7.0 Hz, $10.2 \mathrm{~Hz}), 3.61$ (dd, $1 \mathrm{H}, J=5.3 \mathrm{~Hz}, 10.2 \mathrm{~Hz}), 2.58(\mathrm{~m}, 1 \mathrm{H}), 2.41$ (dt, 1H, $J=7.2 \mathrm{~Hz}, 8.8 \mathrm{~Hz}), 1.96-1.77$ (m, 4H), 1.70-1.59 $(\mathrm{m}, 1 \mathrm{H}), 0.98(\mathrm{~s}, 9 \mathrm{H}), 0.97$ (d, 3H, $J=6.9 \mathrm{~Hz}) ;{ }^{13} \mathrm{C} \mathrm{NMR}\left(100 \mathrm{MHz}, \mathrm{CDCl}_{3}\right) \delta 172.0,135.6,135.5,133.7$, $133.5,129.7,127.7,80.4,65.1,40.4,29.5,26.9,25.5,19.3,18.7,11.1$; HRMS (ESI) calcd. for $\mathrm{C}_{24} \mathrm{H}_{32} \mathrm{O}_{3} \mathrm{NaSi} 419.2018$, found 419.2011, $\Delta=1.6 \mathrm{ppm}$. 


\section{Ketoalcohol 22}

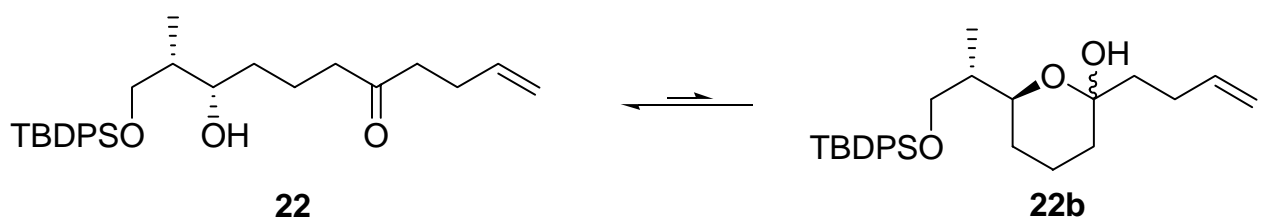

To a stirred mixture of $\mathrm{Mg}$ powder (36 mg, $1.46 \mathrm{mmol}$ ) in THF (3 mL) was added 4-bromo-1-butene $(0.15 \mathrm{~mL}, 1.19 \mathrm{~g}, 1.46 \mathrm{mmol})$. Heat was evolved and the formation of the Grignard reagent was evident from the darkening of the reaction mixture. After $50 \mathrm{~min}, 1.55 \mathrm{~mL}$ of the solution of the Grignard reagent prepared above was added dropwise to a $-78{ }^{\circ} \mathrm{C}$ solution of lactone $21(0.2 \mathrm{~g}, 0.504 \mathrm{mmol})$ in THF $(3 \mathrm{~mL})$. The resulting solution was stirred at $-78{ }^{\circ} \mathrm{C}$ for an additional $40 \mathrm{~min}$ and then saturated aqueous $\mathrm{NH}_{4} \mathrm{Cl}(1.5 \mathrm{~mL})$ and $\mathrm{H}_{2} \mathrm{O}(1.5 \mathrm{~mL})$ were added. The mixture was warmed to $\mathrm{rt}$ and then extracted with MTBE $(3 \times 10 \mathrm{~mL})$. The combined organic phases were dried over $\mathrm{Na}_{2} \mathrm{SO}_{4}$, filtered and concentrated. The crude product was purified twice with flash chromatography (20\% EtOAc in hexanes, 15\% EtOAc in hexanes) to yield ketoalcohol $22(60.7 \mathrm{mg}, 27 \%)$ as a pale yellow oil and lactone 21 (132.8 $\mathrm{mg}, 66 \%)$. This process was repeated twice to obtain 22 in $60 \%$ overall yield.

$R_{f}=0.44\left(40 \%\right.$ EtOAc in hexanes, UV/vanillin); $[\alpha]_{\mathrm{D}}=+0.5\left(c 0.42, \mathrm{CH}_{2} \mathrm{Cl}_{2}\right)$; IR (thin film, $\left.\mathrm{cm}^{-1}\right) 3469$, 2930, 2857, 1713, 1428, 1275, 1261, 1112, 912, 824, 750, 702; ${ }^{1} \mathrm{H}$ NMR (400 MHz, $\left.\mathrm{CDCl}_{3}\right)$ For 22: $\delta$ 7.69-7.66 (m, 4H), 7.48-7.39 (m, 6H), $5.82(\mathrm{ddt}, 1 \mathrm{H}, J=17.1 \mathrm{~Hz}, 10.3 \mathrm{~Hz}, 6.6 \mathrm{~Hz}), 5.01(\mathrm{dq}, 1 \mathrm{H}, J=$ $17.1 \mathrm{~Hz}, 1.8 \mathrm{~Hz}), 4.94(\mathrm{dtd}, 1 \mathrm{H}, J=2.0 \mathrm{~Hz}, 1.3 \mathrm{~Hz}, 10.3 \mathrm{~Hz}), 3.72-3.68$ (m, 1H), 3.67 (dd, 1H, $J=6.7$ $\mathrm{Hz}, 10.0 \mathrm{~Hz}), 3.57$ (dd, 1H, J=5.7 Hz, $9.8 \mathrm{~Hz}), 2.62$ (d, 1H, J=5.5 Hz), 2.49 (t, 2H, J= $7.3 \mathrm{~Hz}), 2.41$ (t, $2 \mathrm{H}, J=7.3 \mathrm{~Hz}), 2.28-2.22(\mathrm{~m}, 2 \mathrm{H}), 1.78-1.27(\mathrm{~m}, 5 \mathrm{H}), 1.03(\mathrm{~s}, 9 \mathrm{H}), 0.84(\mathrm{~d}, 3 \mathrm{H}, J=7.0 \mathrm{~Hz})$; For 22b: $\delta 5.86(\mathrm{~m}), 4.99(\mathrm{dq}, J=17.2 \mathrm{~Hz}, 5.5 \mathrm{~Hz}), 4.90(\mathrm{~m}), 3.56(\mathrm{dd}), 0.95(\mathrm{~d}, 3 \mathrm{H}, J=6.8 \mathrm{~Hz}) ;{ }^{13} \mathrm{C} \mathrm{NMR}(100$ $\mathrm{MHz}, \mathrm{CDCl}_{3}$ ) For 22: $\delta 211.0,136.44,136.40,134.7,134.6,130.8,128.75,115.3,71.9,67.9,43.0,42.2$, 41.4, 34.8, 28.8, 27.2, 21.2, 19.8, 10.9; For 22b: $\delta$ 140.3, 138.7, 130.7, 128.70, 114.5, 96.9, 71.0, 67.0, 43.1, 41.7, 34.0, 28.8, 28.4, 20.0, 12.7; HRMS (ESI) calcd. for $\mathrm{C}_{28} \mathrm{H}_{40} \mathrm{O}_{3} \mathrm{NaSi}$ 475.2644, found 475.2667, $\Delta=4.8 \mathrm{ppm}$.

\section{Ketal 23}

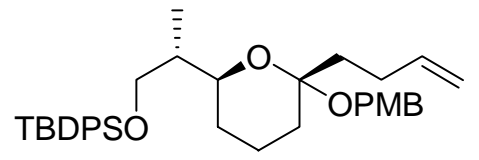

23

To a stirred solution of ketoalcohol $22(50 \mathrm{mg}, 0.11 \mathrm{mmol})$ in $\mathrm{CH}_{2} \mathrm{Cl}_{2}(4 \mathrm{~mL})$ at $\mathrm{rt}$ was added p-methoxybenzyl alcohol $(0.1 \mathrm{~mL}, 0.11 \mathrm{~g}, 0.77 \mathrm{mmol})$ and pyridinium $p$-toluenesulfonate $(5.5 \mathrm{mg}, 0.022$ mmol). The resulting solution was stirred for $3 \mathrm{~h}$ and then saturated aqueous $\mathrm{NaHCO}_{3}(2 \mathrm{~mL})$ and $\mathrm{H}_{2} \mathrm{O}$ $(4 \mathrm{~mL})$ were added. The separated aqueous phase was further extracted with EtOAc $(4 \times 10 \mathrm{~mL})$ and the combined organic phases were dried over $\mathrm{Na}_{2} \mathrm{SO}_{4}$, filtered and concentrated. Silica gel column 
chromatography (3\% MTBE in hexanes, silica gel containing ca. 0.1\% Ca (Fluka)) yielded ketal 23 (53 $\mathrm{mg}, 83 \%)$ as a pale yellow oil.

$R_{f}=0.56\left(60 \%\right.$ EtOAc in hexanes, UV/vanillin); $[\alpha]_{\mathrm{D}}=+7.4\left(\mathrm{c} 1.04, \mathrm{CH}_{2} \mathrm{Cl}_{2}\right)$; IR (thin film, $\left.\mathrm{cm}^{-1}\right) 3070$, 2958, 2931, 2857, 1726, 1472, 1428, 1239, 1112, 1027, 931, 824, 741, 702; $\left.{ }^{1} \mathrm{H} \mathrm{NMR} \mathrm{(400} \mathrm{MHz,} \mathrm{CDCl}_{3}\right)$ $\delta$ 7.65-7.63 (m, 4H), 7.46-7.36 (m, 6H), 7.23-7.20 (m, 2H), 6.82-6.78 (m, 2H), $5.86(\mathrm{ddt}, 1 \mathrm{H}, J=17.1 \mathrm{~Hz}$, $10.3 \mathrm{~Hz}, 6.6 \mathrm{~Hz}), 5.02$ (dq, 1H, $J=17.1 \mathrm{~Hz}, 1.7 \mathrm{~Hz}), 4.93$ (dtd, 1H, J=1.2 Hz, 1.6 Hz, 10.3 Hz), 4.36 (d, $1 \mathrm{H}, J=11.0 \mathrm{~Hz}), 4.27(\mathrm{~d}, 1 \mathrm{H}, J=11.2 \mathrm{~Hz}), 3.78-3.76(\mathrm{~m}, 1 \mathrm{H}), 3.74(\mathrm{~s}, 3 \mathrm{H}), 3.72(\mathrm{dd}, 1 \mathrm{H}, J=5.1 \mathrm{~Hz})$, $3.61(\mathrm{dd}, 1 \mathrm{H}, J=6.0 \mathrm{~Hz}, 10.0 \mathrm{~Hz}), 2.11-2.02(\mathrm{~m}, 2 \mathrm{H}), 1.85-1.16(\mathrm{~m}, 9 \mathrm{H}), 1.01(\mathrm{~d}, 3 \mathrm{H}), 1.00(\mathrm{~s}, 9 \mathrm{H}) ;{ }^{13} \mathrm{C}$ NMR $\left(100 \mathrm{MHz}, \mathrm{CDCl}_{3}\right) \delta 159.9,139.9$ 136.4, 134.84, 134.82, 132.2, 130.71, 130.69, 130.0, 128.7, 114.7, 114.5, 100.4, 71.8, 67.0, 61.9, 55.8, 41.8, 37.3, 33.4, 28.8, 28.7, 27.3, 19.9, 19.8, 12.8; HRMS (ESI) calcd. for $\mathrm{C}_{36} \mathrm{H}_{48} \mathrm{O}_{4} \mathrm{NaSi} 595.3220$, found 595.3209, $\Delta=1.8 \mathrm{ppm}$.

\section{Dihydroxyketal 24}

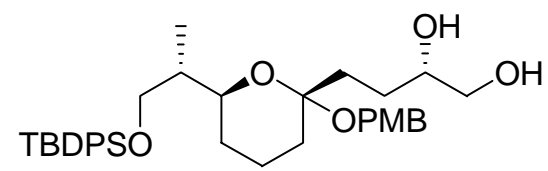

24a

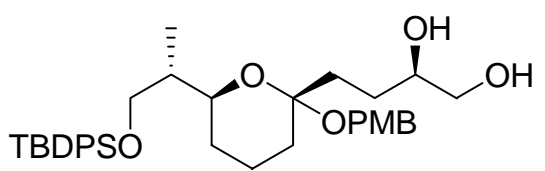

24b

(DHQ) 2 PYR (5.6 mg, $0.006 \mathrm{mmol}), \mathrm{K}_{3} \mathrm{Fe}(\mathrm{CN})_{6}(89 \mathrm{mg}, 0.27 \mathrm{mmol}), \mathrm{K}_{2} \mathrm{CO}_{3}(37 \mathrm{mg}, 0.27 \mathrm{mmol})$, $\mathrm{CH}_{3} \mathrm{SO}_{2} \mathrm{NH}_{2}(8.6 \mathrm{mg}, 0.09 \mathrm{mmol})$ and $\mathrm{K}_{2} \mathrm{OsO}_{4} \cdot 2 \mathrm{H}_{2} \mathrm{O}(0.3 \mathrm{mg}, 0.0009 \mathrm{mmol})$ were dissolved in 1:1 tert-butyl alcohol/water $(1.5 \mathrm{~mL}$ each) at $\mathrm{rt}$. The resulting mixture was vigorously stirred for $20 \mathrm{~min}$ and then cooled to $0{ }^{\circ} \mathrm{C}$. A solution of ketal $23(52 \mathrm{mg}, 0.09 \mathrm{mmol})$ in tert-butyl alcohol $(0.5 \mathrm{~mL})$ was added via cannula. Stirring was continued at $0{ }^{\circ} \mathrm{C}$ for an additional $17.5 \mathrm{~h}$ before $\mathrm{Na}_{2} \mathrm{SO}_{3}(0.14 \mathrm{~g})$ was added. The resulting mixture was vigorously stirred and allowed to warm to rt. The mixture was diluted with EtOAc $(10 \mathrm{~mL})$. Separated aqueous phase was extracted with EtOAc $(3 \times 5 \mathrm{~mL})$ and the combined organic phases were washed with $\mathrm{H}_{2} \mathrm{O}(10 \mathrm{~mL})$, dried over $\mathrm{Na}_{2} \mathrm{SO}_{4}$, filtered and concentrated. The crude product was immediately used in the next reaction without further purification.

$R_{f}=0.16\left(60 \%\right.$ EtOAc in hexanes, UV/vanillin); ${ }^{1} \mathrm{H}$ NMR $\left(400 \mathrm{MHz}, \mathrm{CDCl}_{3}\right) \delta 7.65-7.63(\mathrm{~m}, 4 \mathrm{H}), 7.47-$ 7.37 (m, 6H), 7.23-7.20 (m, 2H), 6.81-6.79 (m, 2H), 4.36-4.26 (m, 2H), 3.77-3.76 (m, 1H), $3.74(\mathrm{~s}, 3 \mathrm{H})$, $3.72(\mathrm{dd}, 1 \mathrm{H}, J=5.0 \mathrm{~Hz}), 3.61(\mathrm{dd}, 1 \mathrm{H}, J=5.8 \mathrm{~Hz}, 9.9 \mathrm{~Hz}), 3.49(\mathrm{~m}, 1 \mathrm{H}), 3.41(\mathrm{~m}, 1 \mathrm{H}), 3.30(\mathrm{~m}, 1 \mathrm{H})$, $2.82(\mathrm{~m}, 1 \mathrm{H}), 2.69(\mathrm{~m}, 1 \mathrm{H}), 1.81-1.23(\mathrm{~m}, 11 \mathrm{H}), 1.01(\mathrm{~d}, 3 \mathrm{H}), 1.00(\mathrm{~s}, 9 \mathrm{H}) ;{ }^{13} \mathrm{C} \mathrm{NMR}\left(100 \mathrm{MHz}, \mathrm{CDCl}_{3}\right)$ $\delta 159.8,136.4,134.82,134.80,132.3,130.69,130.68,130.0,128.7,114.5,100.5,72.8,71.8,67.1,67.0$, $61.8,55.8,41.8,34.1,33.4,28.8,28.2,27.3,19.9,19.8,12.8$; For $\mathbf{2 4 b}$, the following signals were also observed in ${ }^{13} \mathrm{C}$ NMR: $\delta 72.8,67.1,61.9,34.2,28.9,28.1$. 


\section{Pivalate 25}

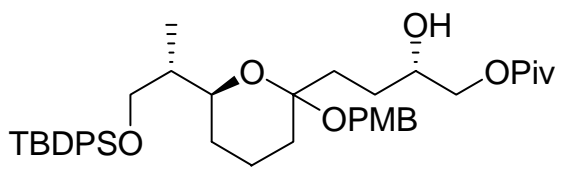

$25 a$

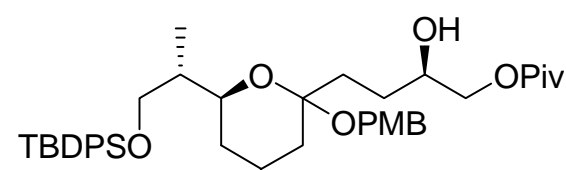

25b

To a stirred $0{ }^{\circ} \mathrm{C}$ solution of dihydroxyketal $24(0.05 \mathrm{~g}, 0.082 \mathrm{mmol})$ in pyridine $(0.5 \mathrm{~mL})$ was added pivaloyl chloride $(12 \mu 1,12 \mathrm{mg}, 0.1 \mathrm{mmol})$. The resulting solution was stirred at $0{ }^{\circ} \mathrm{C}$ for an additional $2 \mathrm{~h}$ $15 \mathrm{~min}$. Aqueous saturated $\mathrm{NaHCO}_{3}(1 \mathrm{~mL})$ and $\mathrm{H}_{2} \mathrm{O}(5 \mathrm{~mL})$ were added and the phases were separated. The aqueous phase was extracted with EtOAc $(3 \times 5 \mathrm{~mL})$ and the combined organic phases were washed with $\mathrm{H}_{2} \mathrm{O}(5 \mathrm{~mL})$, dried over $\mathrm{Na}_{2} \mathrm{SO}_{4}$, filtered and concentrated. Silica gel column chromatography (15\% EtOAc in hexanes) yielded pivalate 25 (40.1 mg, 62\% over 2 steps) as a pale yellow viscous oil.

$R_{f}=0.47$ (40\% EtOAc in hexanes, UV/vanillin); $[\alpha]_{\mathrm{D}}=+12.4\left(\mathrm{c} 0.27, \mathrm{CH}_{2} \mathrm{Cl}_{2}\right)$; IR (thin film, $\left.\mathrm{cm}^{-1}\right) 3469$, 2930, 2957, 1713, 1428, 1275, 1261, 1112, 912, 824, 750, 701; $1^{\mathrm{H}} \mathrm{NMR}\left(400 \mathrm{MHz}, \mathrm{CDCl}_{3}\right)$ For 25a: $\delta$ 7.65-7.62 (m, 4H), 7.46-7.36 (m, 6H), 7.22-7.20 (m, 2H), 6.80-6.78 (m, 2H), 4.35 (dd, 1H, J = 3.0 Hz, $11.1 \mathrm{~Hz}), 4.28$ (dd, 1H, J=4.6 Hz, $11.1 \mathrm{~Hz}), 3.96-3.91$ (m, 2H), 3.77-3.76 (m, 1H), 3.74 (s, 3H), 3.72 $(\mathrm{dd}, 1 \mathrm{H}, J=4.9 \mathrm{~Hz}), 3.61(\mathrm{dd}, 1 \mathrm{H}, J=5.9 \mathrm{~Hz}, 10.0 \mathrm{~Hz}), 2.97(\mathrm{~d}, 1 \mathrm{H}, J=5.5 \mathrm{~Hz}), 1.80-1.21(\mathrm{~m}, 11 \mathrm{H})$, $1.17(\mathrm{~s}, 9 \mathrm{H}), 1.01(\mathrm{dd}, 3 \mathrm{H}), 1.00(\mathrm{~s}, 9 \mathrm{H})$; For $25 \mathrm{~b}$, the following additional resonances could be observed: $\delta 2.97(\mathrm{~d}, J=5.3 \mathrm{~Hz}), 1.17(\mathrm{~s}) ;{ }^{13} \mathrm{C} \mathrm{NMR}\left(100 \mathrm{MHz}, \mathrm{CDCl}_{3}\right)$ For 25a: $\delta 178.93,159.8,136.4,134.83$, 134.81, 132.3, 130.70, 130.69, 130.0, 129.9, 128.7, 114.5, 100.4, 71.8, 70.1, 68.9, 67.0, 61.85, 55.8, 41.9, $39.4,34.0,33.4,28.8,28.4,27.4,27.2,19.9,19.8,12.8$; For 25b, the following additional resonances could be observed: $\delta 178.91,100.5,70.0,68.8,61.89$, 28.5; HRMS (ESI) calcd. for $\mathrm{C}_{41} \mathrm{H}_{58} \mathrm{O}_{7} \mathrm{NaSi}$ 713.3850 , found $713.3872, \Delta=3.0 \mathrm{ppm}$.

\section{Spiroketals 12 and 26}

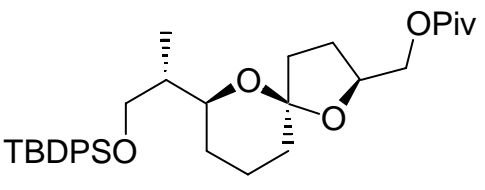

12

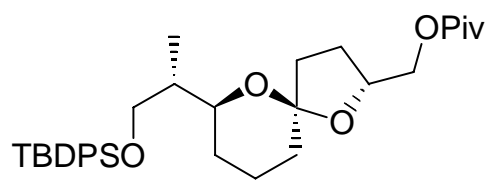

26

To a solution of pivalate $25(3.5 \mathrm{mg}, 0.005 \mathrm{mmol})$ in $\mathrm{CDCl}_{3}(1 \mathrm{~mL})$ was added $\mathrm{AcOH}$ (1 drop, ca $10 \mu \mathrm{L}$, $3300 \mathrm{~mol} \%$ ) at rt. After $21 \mathrm{~h}$, the reaction was quenched by the addition of saturated aqueous $\mathrm{NaHCO}_{3}$ $(0.5 \mathrm{~mL})$ and $\mathrm{H}_{2} \mathrm{O}(0.5 \mathrm{~mL})$. The separated aqueous phase was extracted with EtOAc $(3 \times 3 \mathrm{~mL})$ and the combined organic phases were dried over $\mathrm{Na}_{2} \mathrm{SO}_{4}$, filtered and concentrated. Silica gel column chromatography (3\% EtOAc in hexanes) yielded fraction A: spiroketal 12 (1.5 mg, 54\%), fraction B: a mixture of spiroketals 12 and 26 (0.5 mg, 17\%), fraction C: spiroketal 26 (0.8 mg, 29\%). 
12: $R_{f}=0.33\left(10 \%\right.$ EtOAc in hexanes, UV/vanillin); $[\alpha]_{\mathrm{D}}=+2.6\left(\mathrm{c} 0.53, \mathrm{CH}_{2} \mathrm{Cl}_{2}\right)$; IR (thin film, $\left.\mathrm{cm}^{-1}\right)$ 2930, 2857, 1732, 1460, 1367, 1282, 1153, 1113, 1075, 1023, 824, 742, 702; ${ }^{1} \mathrm{H}$ NMR $\left(400 \mathrm{MHz}, \mathrm{CDCl}_{3}\right)$ $\delta$ 7.67-7.64 (m, 4H), 7.48-7.38 (m, 6H), 4.19-4.12 (m, 1H), 4.03 (dd, 1H, J = 3.9 Hz, 11.4 Hz), 3.97 (dd, $1 \mathrm{H}, J=5.0 \mathrm{~Hz}, 11.4 \mathrm{~Hz}$ ), $3.83(\mathrm{ddd}, 1 \mathrm{H}, J=2.1 \mathrm{~Hz}, 5.3 \mathrm{~Hz}, 11.7 \mathrm{~Hz}), 3.66(\mathrm{dd}, 1 \mathrm{H}, J=6.0 \mathrm{~Hz}, 9.9 \mathrm{~Hz}$ ), $3.52(\mathrm{dd}, 1 \mathrm{H}, J=6.0 \mathrm{~Hz}, 9.9 \mathrm{~Hz}), 2.05-1.98(\mathrm{~m}, 1 \mathrm{H}), 1.79-1.57$ (m, 7H), 1.49-1.44 (m, 1H), 1.36-1.22 (m, 2H), 1.15 (s, 9H), 1.04 (s, 9H), 0,90 (d, 3H, $J=7.0 \mathrm{~Hz}$ ); ${ }^{13} \mathrm{C}$ NMR (100 MHz, $\left.\mathrm{CDCl}_{3}\right) \delta 178.8,136.41$, 136.39, 134.89, 134.84, 130.7, 128.7, 107.2, 76.3, 71.4, 67.0, 66.7, 41.4, 39.4, 38.1, 33.5, 28.7, 27.4, 27.3, 26.6, 21.4, 19.8, 12.4; HRMS (ESI) calcd. for $\mathrm{C}_{33} \mathrm{H}_{48} \mathrm{O}_{5} \mathrm{NaSi} 575.3169$, found 575.3176, $\Delta=1.2 \mathrm{ppm}$.

26: $R_{f}=0.30\left(10 \%\right.$ EtOAc in hexanes, UV/vanillin); $[\alpha]_{\mathrm{D}}=+1.4\left(c 0.14, \mathrm{CH}_{2} \mathrm{Cl}_{2}\right) ;{ }^{1} \mathrm{H}$ NMR $(400 \mathrm{MHz}$, $\left.\mathrm{CDCl}_{3}\right) \delta$ 7.67-7.64 (m, 4H), 7.47-7.38 (m, 6H), 4.21- $4.13(\mathrm{~m}, 1 \mathrm{H}), 3.99$ (dd, 1H, $\left.J=7.3 \mathrm{~Hz}, 11.0 \mathrm{~Hz}\right)$, $3.94(\mathrm{dd}, 1 \mathrm{H}, J=5.3 \mathrm{~Hz}, 11.0 \mathrm{~Hz}), 3.73(\mathrm{ddd}, 1 \mathrm{H}, J=2.1 \mathrm{~Hz}, 5.7 \mathrm{~Hz}), 3.70(\mathrm{dd}, 1 \mathrm{H}, J=4.6 \mathrm{~Hz}, 9.9 \mathrm{~Hz})$, 3.54 (dd, 1H, $J=6.9 \mathrm{~Hz}, 9.9 \mathrm{~Hz}), 2.11-2.10(\mathrm{~m}, 1 \mathrm{H}), 1.86-1.47$ (m, 10H), 1.15 (s, 9H), 1.03 (s, 9H), 0,97 $(\mathrm{d}, 3 \mathrm{H}, J=6.8 \mathrm{~Hz}) ;{ }^{13} \mathrm{C}$ NMR $\left(100 \mathrm{MHz}, \mathrm{CDCl}_{3}\right) \delta 178.7,136.4,134.9,134.8,130.69,130.67,128.7$, $107.3,78.3,72.2,69.0,67.1,41.9,39.0$ (2 C), 34.1, 28.6, 27.8, 27.4, 27.3, 21.2, 19.8, 13.4. HRMS (ESI) calcd. for $\mathrm{C}_{33} \mathrm{H}_{48} \mathrm{O}_{5} \mathrm{NaSi}$ 575.3169, found 575.3157, $\Delta=2.1 \mathrm{ppm}$.

\section{Spiroketal 11}

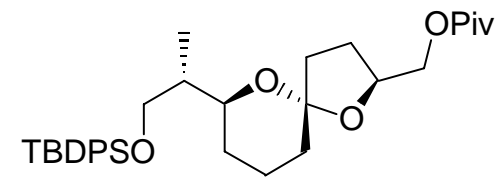

11

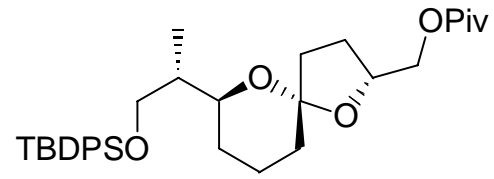

27

To a solution of pivalate $25(10.0 \mathrm{mg}, 0.014 \mathrm{mmol})$ in $\mathrm{CH}_{2} \mathrm{Cl}_{2}(1 \mathrm{~mL})$ was added chloroacetic acid $(0.27$ $\mathrm{mg}, 0.0029 \mathrm{mmol})$ in $\mathrm{CH}_{2} \mathrm{Cl}_{2}(0.8 \mathrm{ml})$ at $\mathrm{rt}$. After $30 \mathrm{~min}$, another portion of chloroacetic acid $(0.27 \mathrm{mg}$, $0.0029 \mathrm{mmol})$ in $\mathrm{CH}_{2} \mathrm{Cl}_{2}(0.8 \mathrm{ml})$ was added. The resulting solution was stirred at $\mathrm{rt}$ for an additional $4 \mathrm{~h}$. The reaction was quenched by addition of saturated aqueous $\mathrm{NaHCO}_{3}(0.5 \mathrm{~mL})$ and $\mathrm{H}_{2} \mathrm{O}(0.5 \mathrm{~mL})$. The separated aqueous phase was extracted with EtOAc $(3 \times 5 \mathrm{~mL})$ and the combined organic phases were dried over $\mathrm{Na}_{2} \mathrm{SO}_{4}$, filtered and concentrated. Silica gel column chromatography (3\% EtOAc in hexanes) yielded fraction A: spiroketal $12(1.9 \mathrm{mg}, 24 \%)$, fraction B: a mixture of spiroketals 12 and 26 (1.6 mg, 21\%), fraction C: spiroketal 26 ( $0.5 \mathrm{mg}, 6 \%$ ), fraction D: spiroketal 11 (containing ca $5 \%$ of 27) (3.8 mg, 49\%).

Fraction D: $R_{f}=0.20\left(10 \%\right.$ EtOAc in hexanes, UV/vanillin); $[\alpha]_{\mathrm{D}}=+5.4\left(\mathrm{c} 0.28, \mathrm{CH}_{2} \mathrm{Cl}_{2}\right)$; IR (thin film, $\left.\mathrm{cm}^{-1}\right)$ 2929, 2857, 1732, 1463, 1277, 1263, 1156, 1113, 1009, 896, 824, 748, 703; ${ }^{1} \mathrm{H}$ NMR (400 MHz, $\left.\mathrm{CDCl}_{3}\right) \delta$ 7.68-7.65 (m, 4H), 7.48-7.39 (m, 6H), 4.20-4.14 (dt, $\left.J=4.6 \mathrm{~Hz}, 7.3 \mathrm{~Hz}\right), 4.02(\mathrm{dd}, 1 \mathrm{H}, J=4.6$ $\mathrm{Hz}, 11.1 \mathrm{~Hz}), 3.92(\mathrm{dd}, 1 \mathrm{H}, J=7.3 \mathrm{~Hz}, 11.1 \mathrm{~Hz}), 3.67$ (dd, $1 \mathrm{H}, J=6.8 \mathrm{~Hz}, 9.9 \mathrm{~Hz}), 3.63-3.58$ (ddd, $1 \mathrm{H}, J$ $=2.3 \mathrm{~Hz}, 4.5 \mathrm{~Hz}, 11.5 \mathrm{~Hz}), 3.52(\mathrm{dd}, 1 \mathrm{H}, J=6.0 \mathrm{~Hz}, 9.9 \mathrm{~Hz}), 2.42-2.37(\mathrm{~m}, 1 \mathrm{H}), 1.79-1.24(\mathrm{~m}, 10 \mathrm{H})$, 1.17 (s, 9H), 1.04 (s, 9H), 0.89 (d, 3H, $J=6.9 \mathrm{~Hz}) ;{ }^{13} \mathrm{C}$ NMR (100 MHz, $\left.\mathrm{CDCl}_{3}\right) \delta$ 178.7, 136.4, 134.8, 
$130.7,128.7,109.4,78.1,75.0,69.2,67.0,41.4,39.3,34.8,32.2,28.9,27.6,27.4,27.2,22.9,19.8,12.3$; HRMS (ESI) calcd. for $\mathrm{C}_{33} \mathrm{H}_{48} \mathrm{O}_{5} \mathrm{NaSi} 575.3169$, found 575.3159, $\Delta=1.7 \mathrm{ppm}$.

Additional evidence for the assigned nonanomeric structure of 11 (Figure 3). ${ }^{1} \mathrm{H}$ NMR coupling constant data clearly indicates a C4 chair conformation for the A ring.

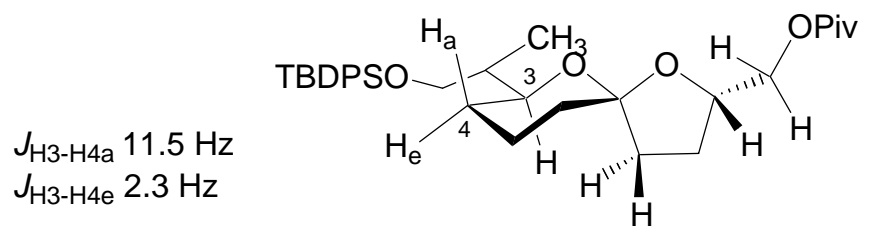

11

Spiroketalization with PPTS (Table 1, entry 1) afforded 11 accompanied by ca. $10 \%$ of the inseparable $\mathrm{C}_{10}$ epimer 27. For spiroketal 27, the following additional resonances could be observed: $\delta 4.29-4.22(\mathrm{~m})$, $4.05(\mathrm{dd}, J=3.8 \mathrm{~Hz}), 3.95(\mathrm{dd}, J=5.1 \mathrm{~Hz}), 3.69-3.65(\mathrm{~m}), 1.18(\mathrm{~s}), 0.89$ (d, $J=6.8 \mathrm{~Hz})$.

\section{References}

1. Gao, Y.; Hanson, R. M.; Klunder, J. M.; Ko, S. Y.; Masamune, H.; Sharpless, K. B. J. Am. Chem. Soc. 1987, 109, 5765-5780.

2. Börjesson, L.; Csöregh, I.; Welch, C. J. J. Org. Chem. 1995, 60, 2989-2999.

3. Nicolaou, K. C.; Veale, C. A.; Webber, S. E.; Katerinopoulos, H. J. Am. Chem. Soc. 1985, 107, 75157518 . 
7-(Benzyloxy)-methyl-2-(Z)-heptenoate 16
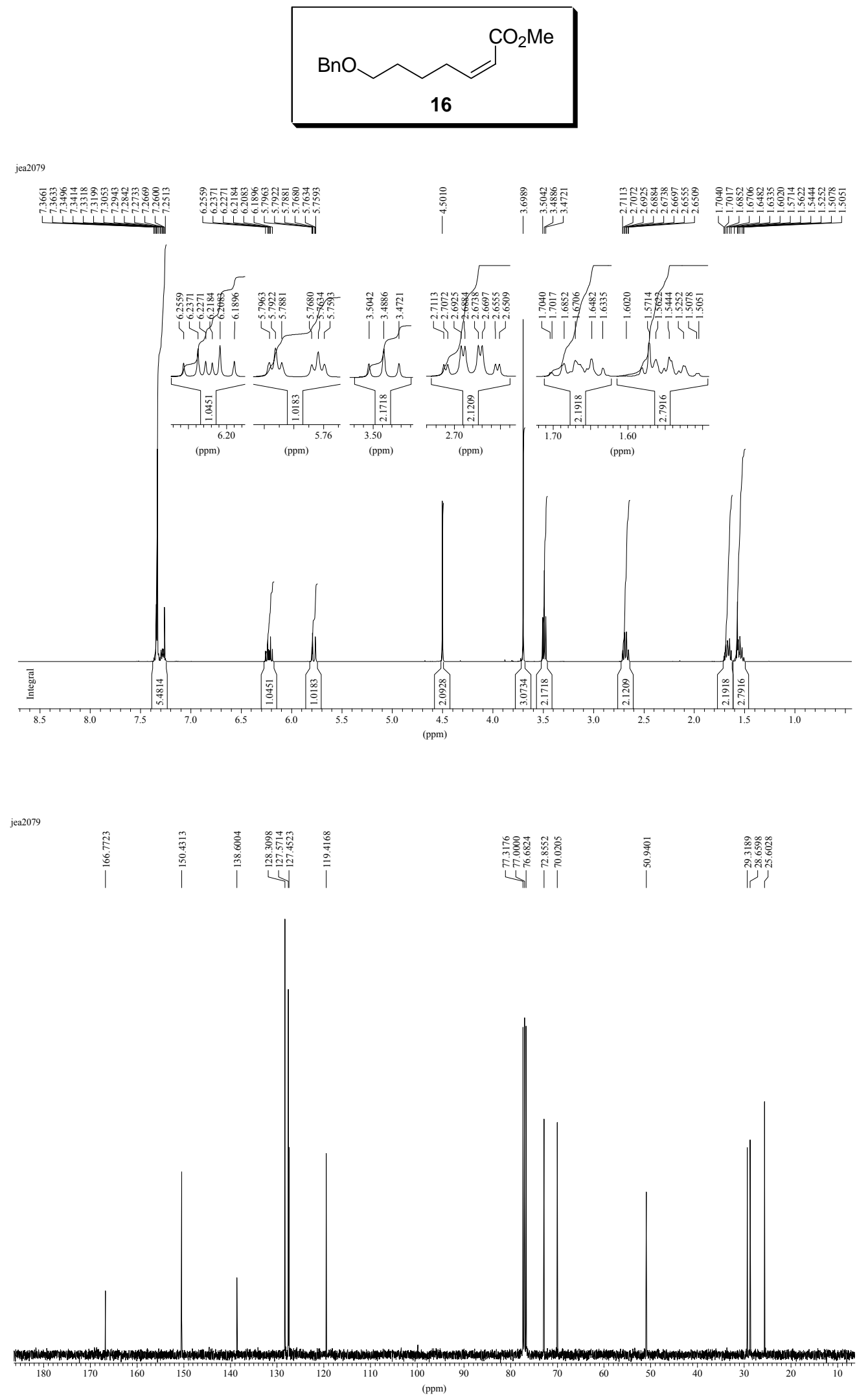
7-(Benzyloxy)-methyl-2-(Z)-hepten-1-ol 17

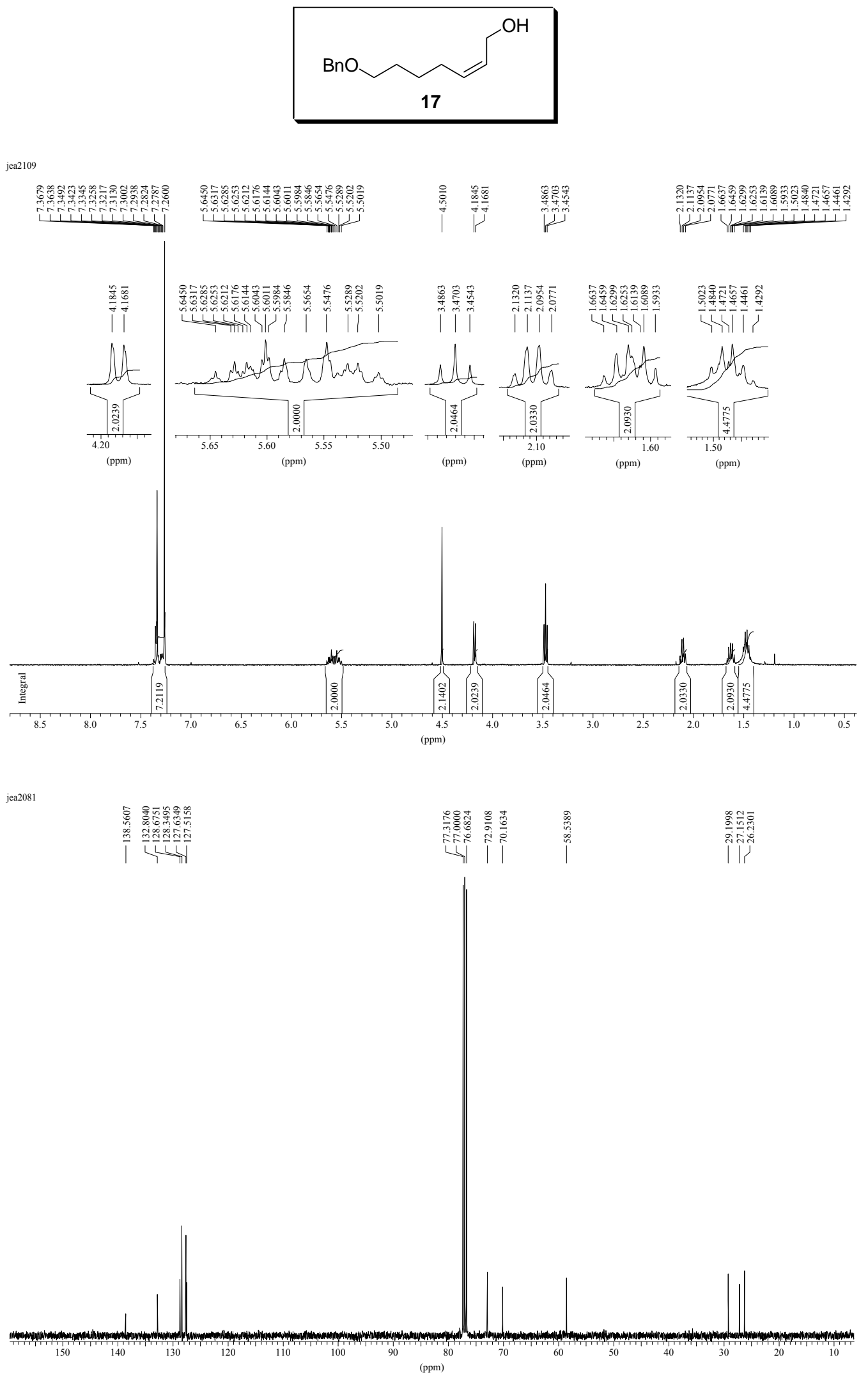




\section{Epoxide 18}

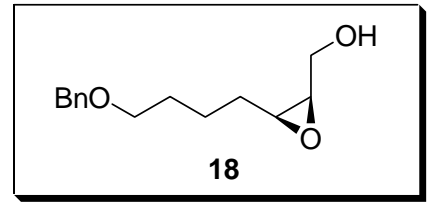

jea2095

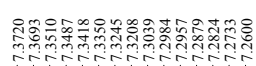

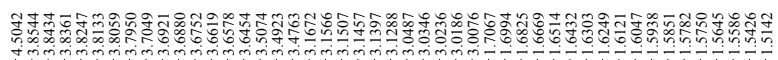

का

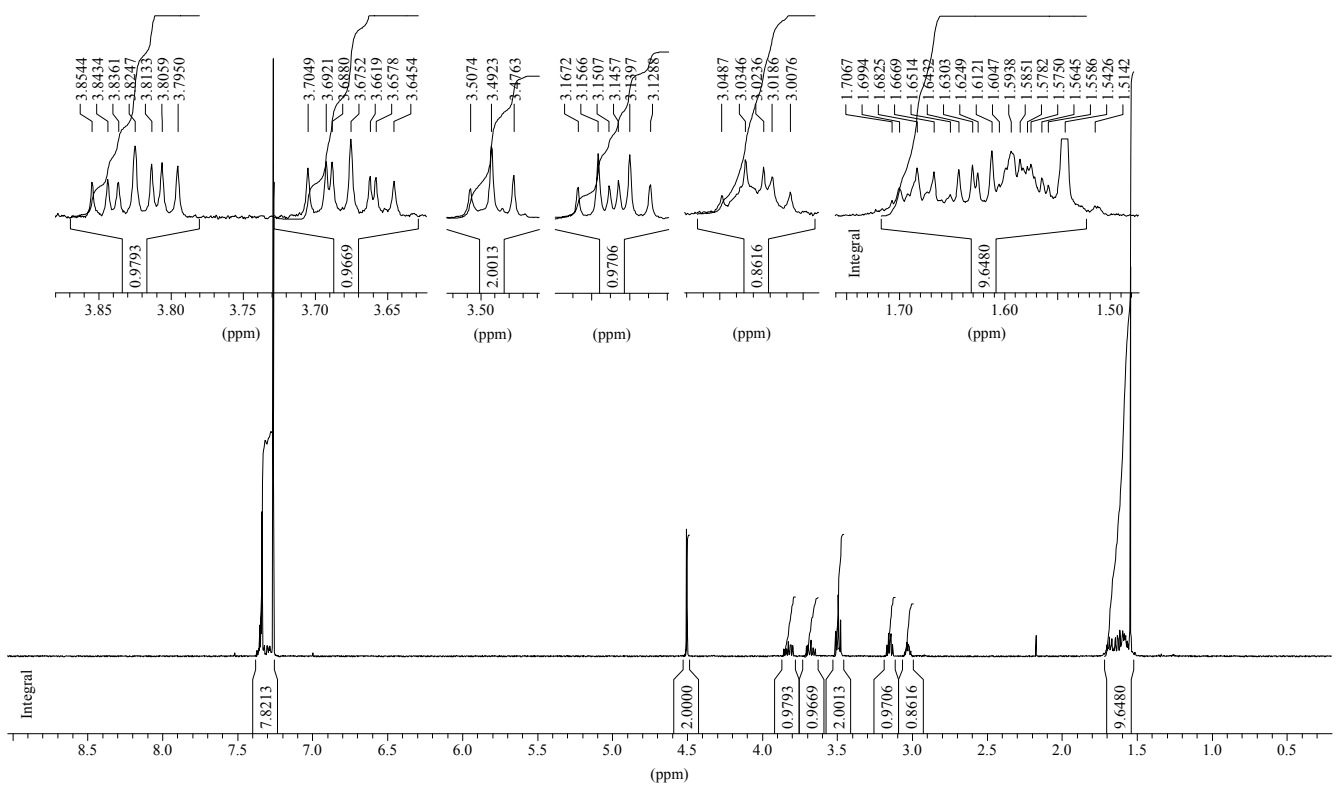

jea208:

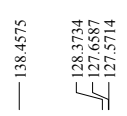

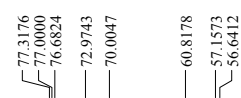

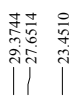

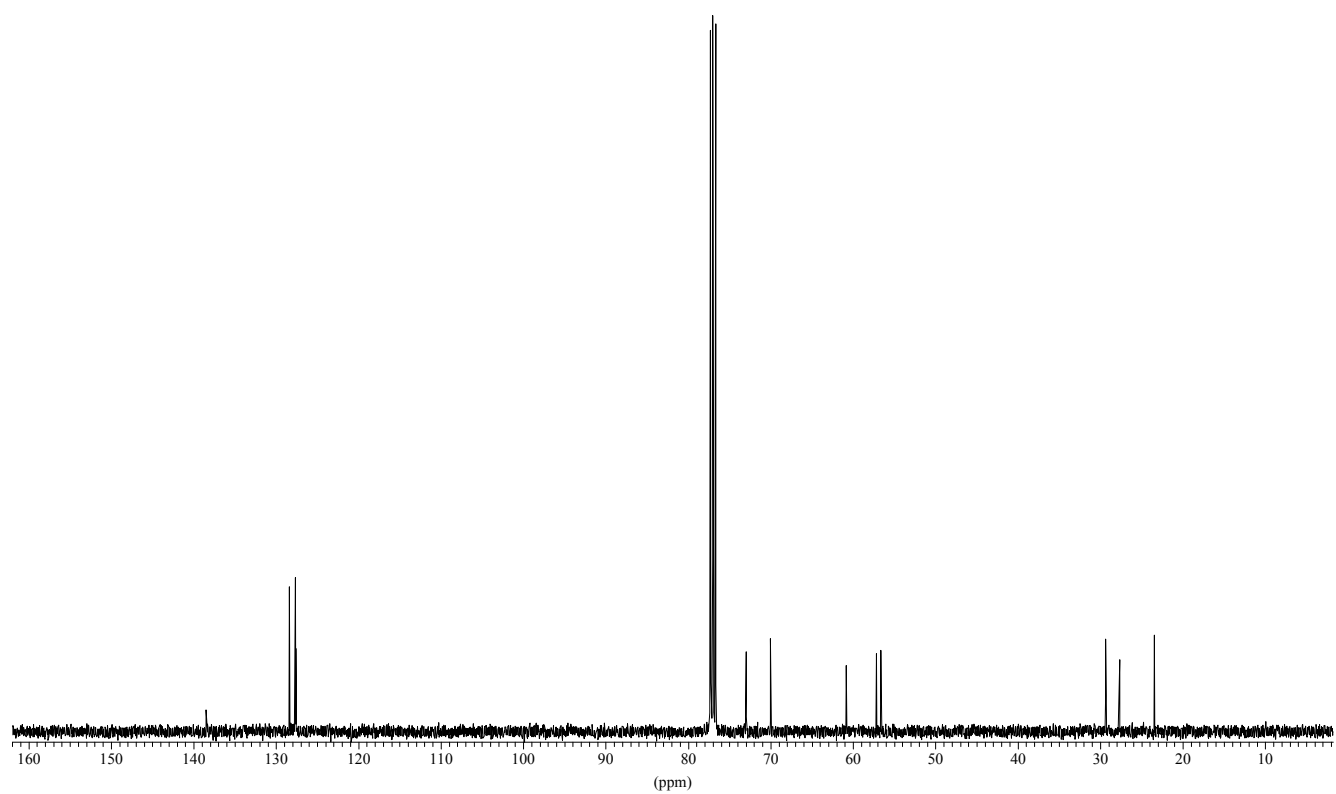


7-(Benzyloxy)-2-methyl-heptane-1,3-diol 19
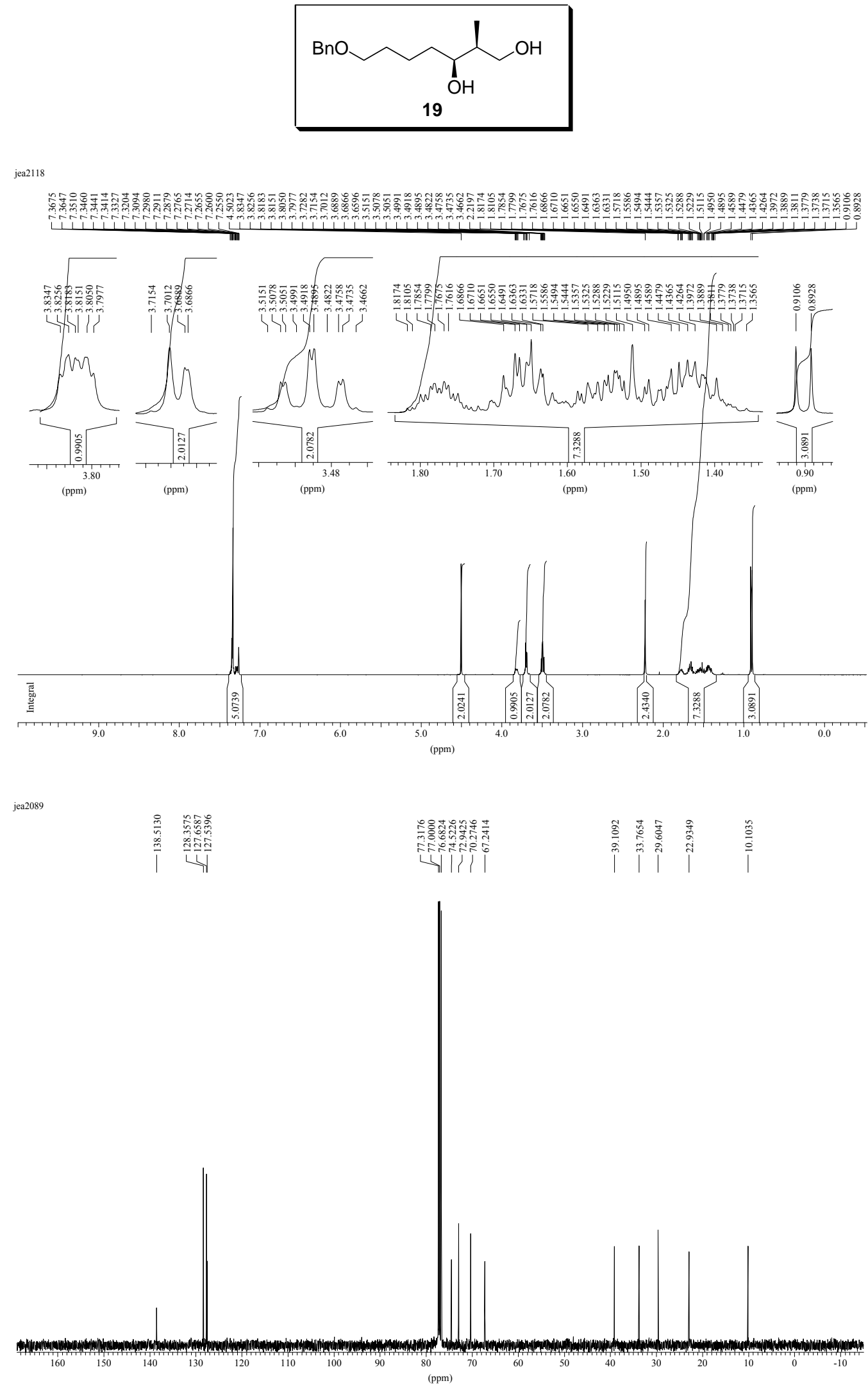
7-(Benzyloxy)-2-methyl-1-(t-butyl-diphenylsilyl)-heptan-3-ol
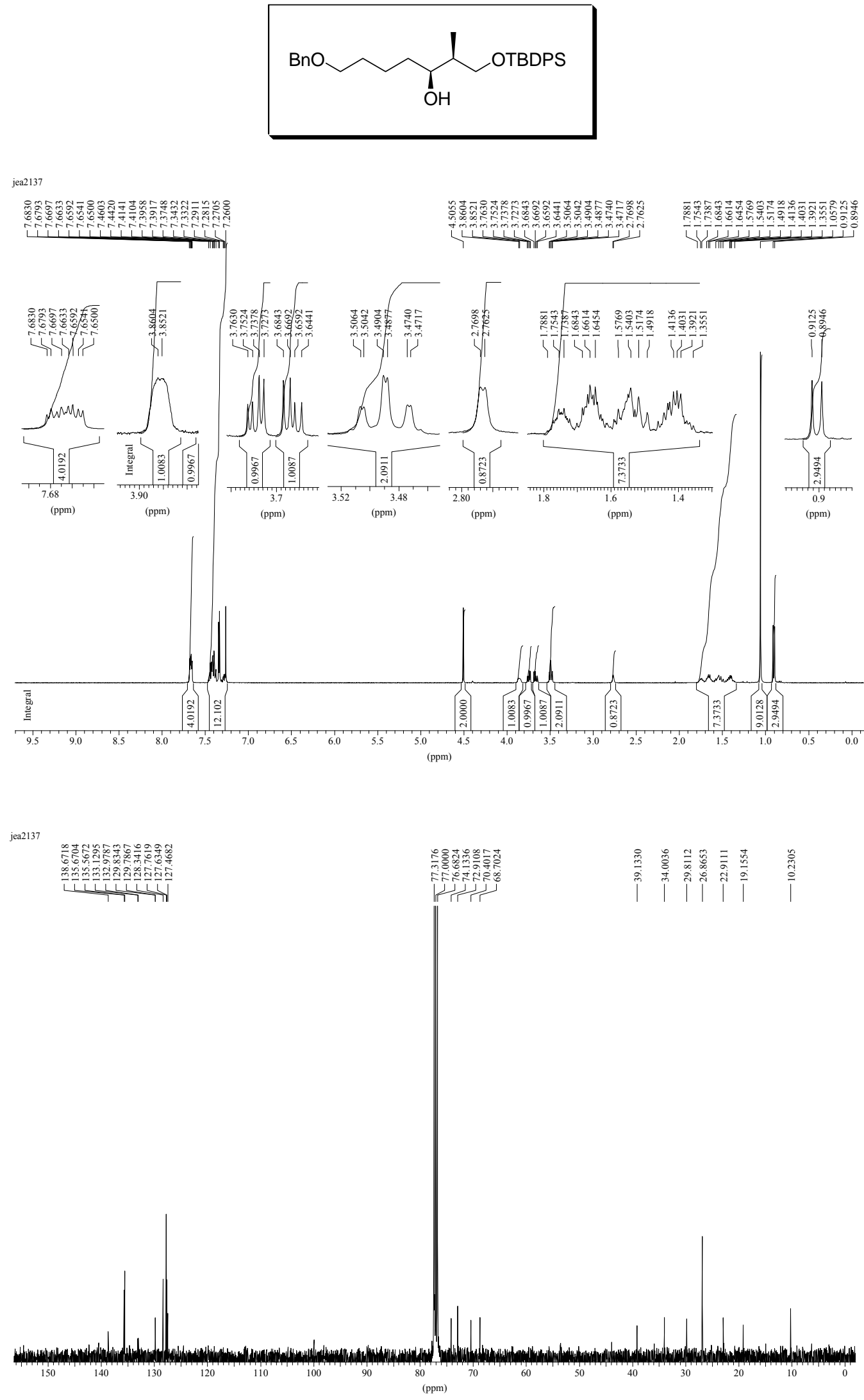
6-Methyl-7-(t-butyl-diphenylsilyl)-heptane-1,5-diol 20
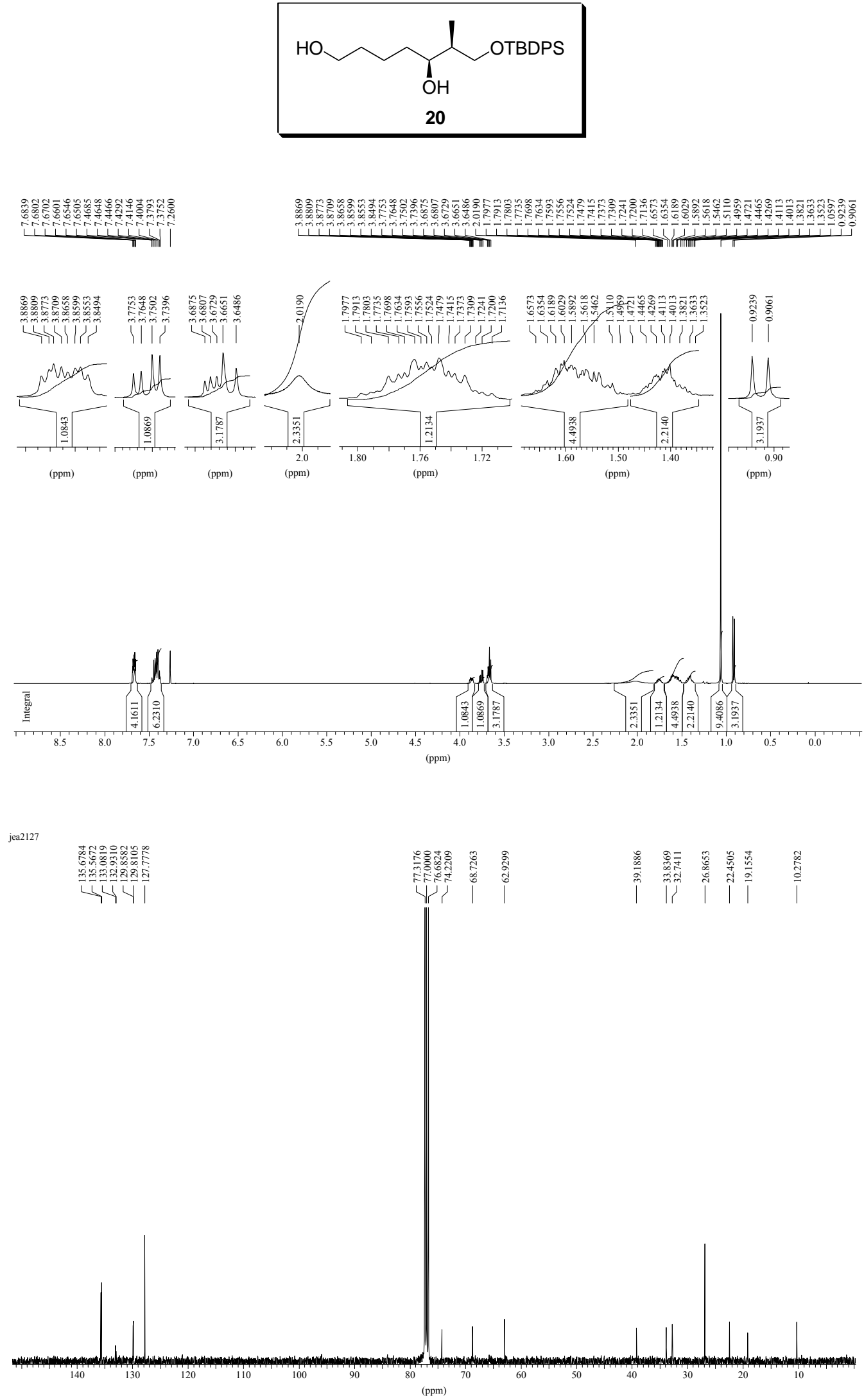
6-Methyl-7-(t-butyl-diphenylsilyl)-heptane-1,5-diol 20
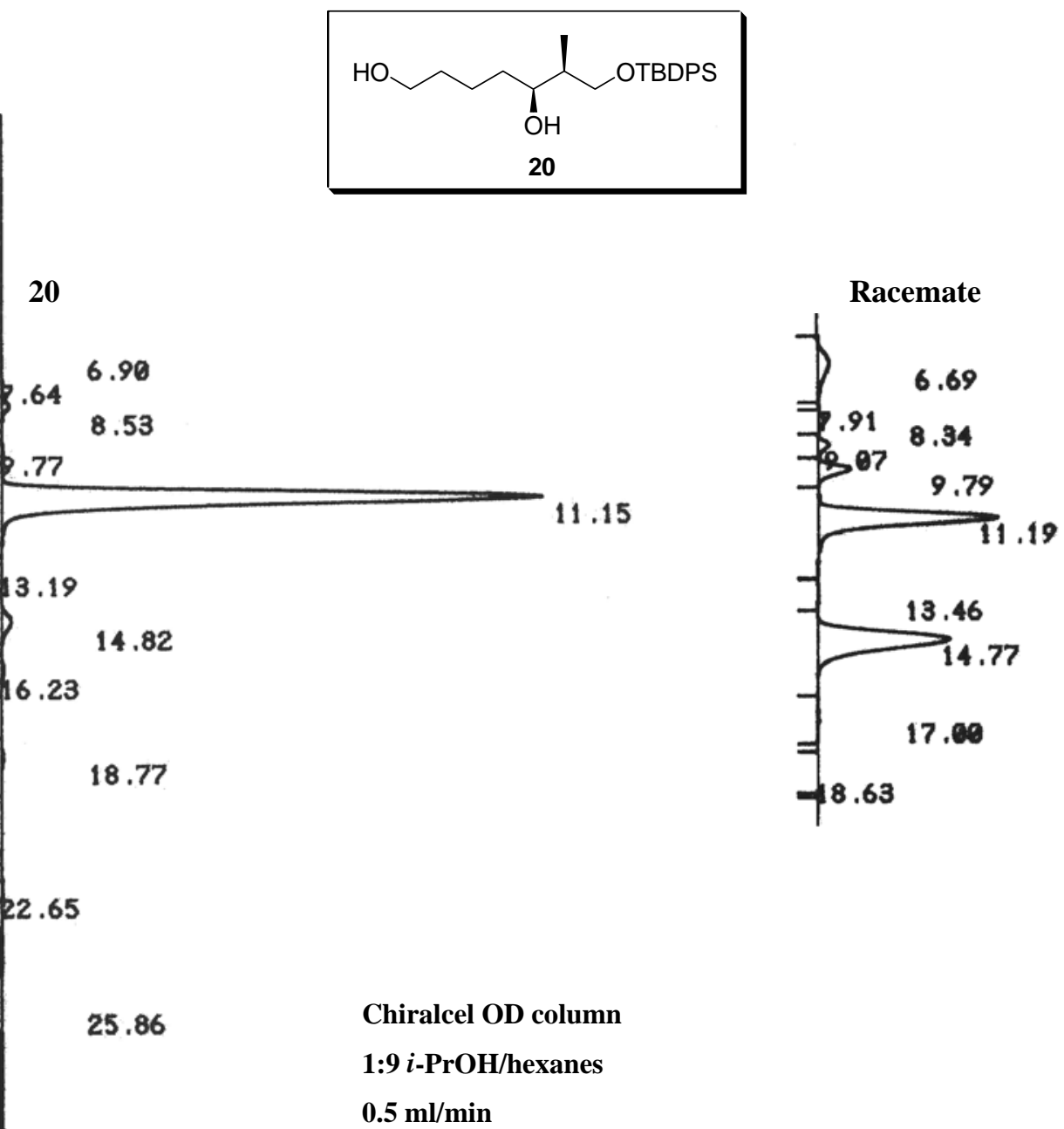

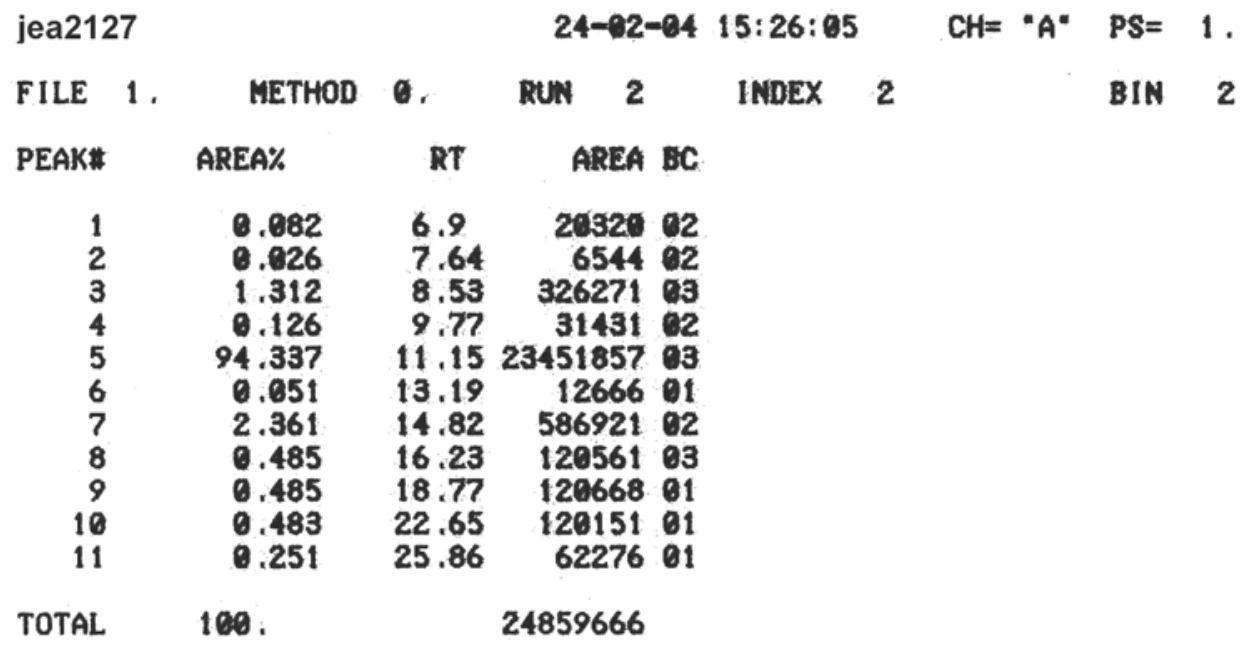




\section{Lactone 21}

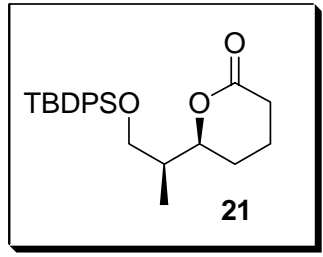

jea2133
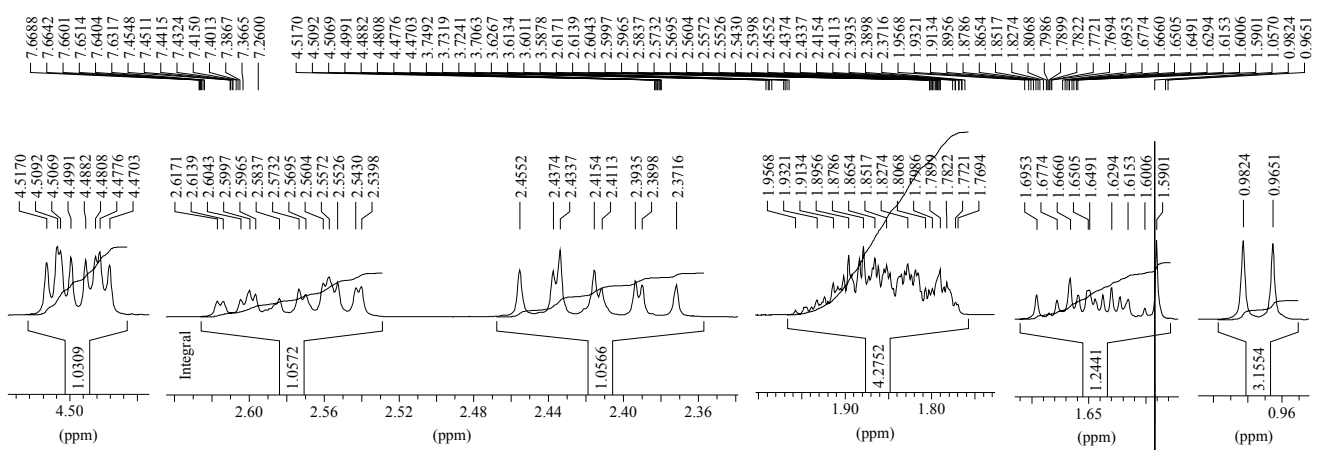

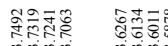
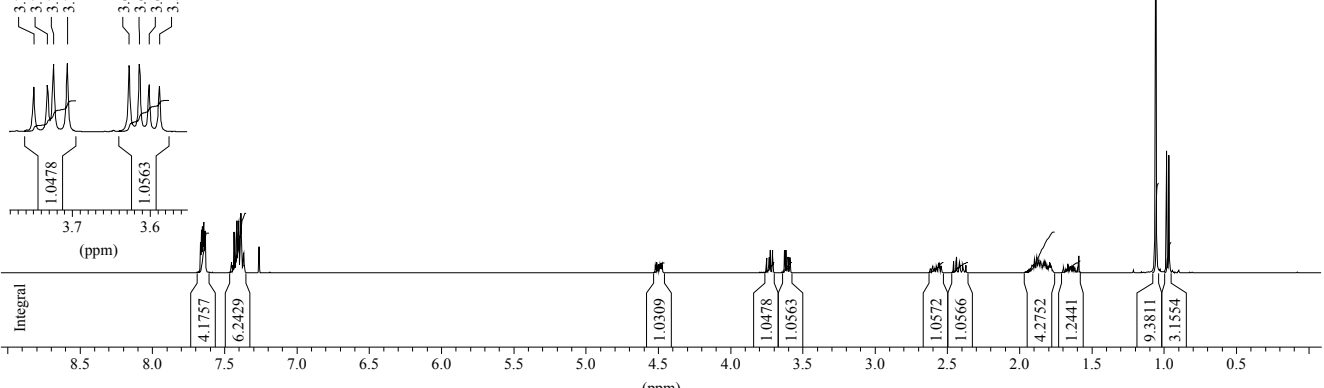

jea2133
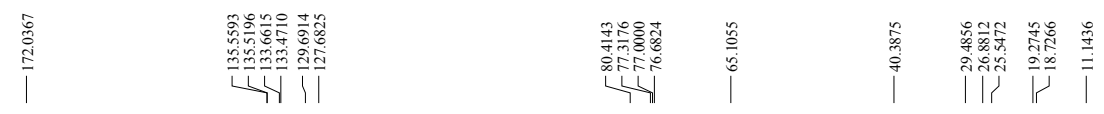

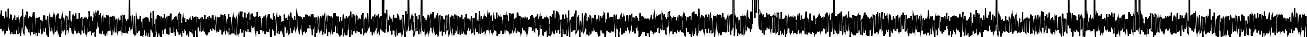

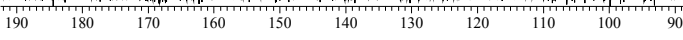




\section{Keto alcohol 22}

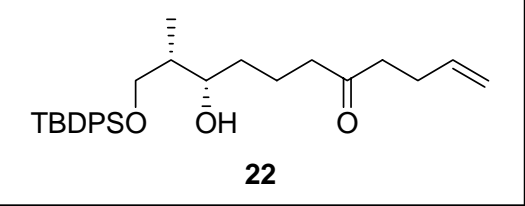

jea2140

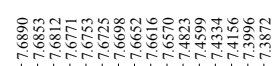

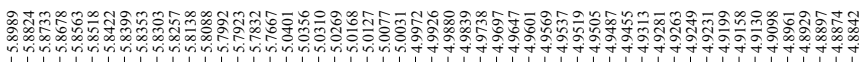

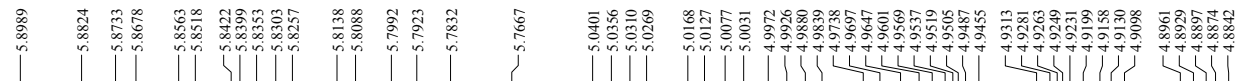
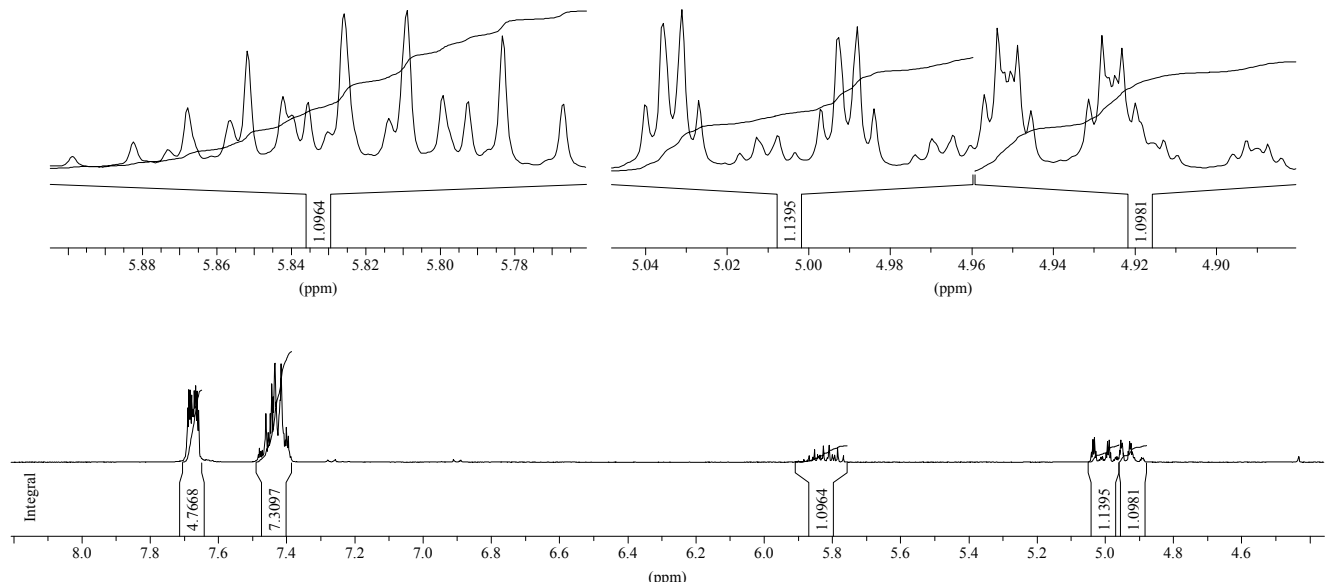

jea 2140

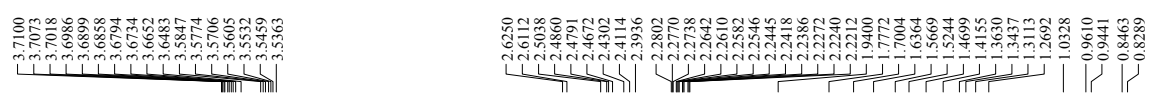

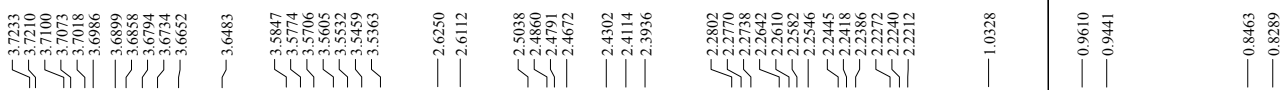
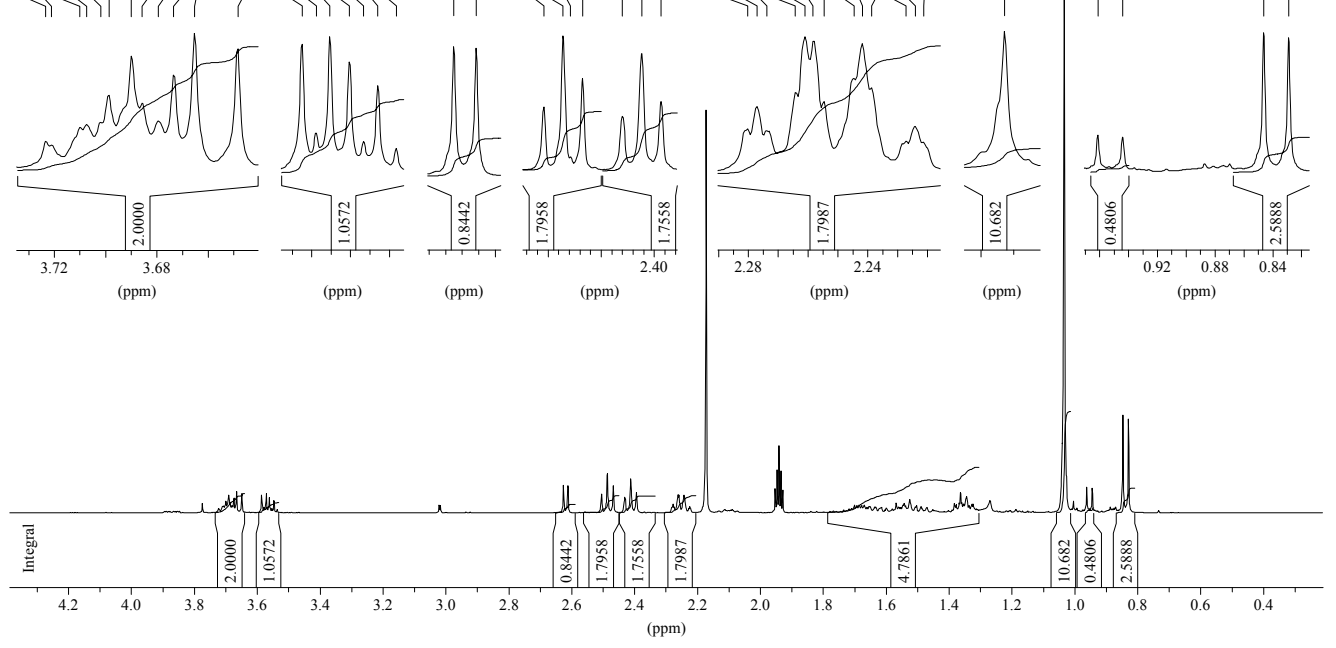
Ketoalcohol 22
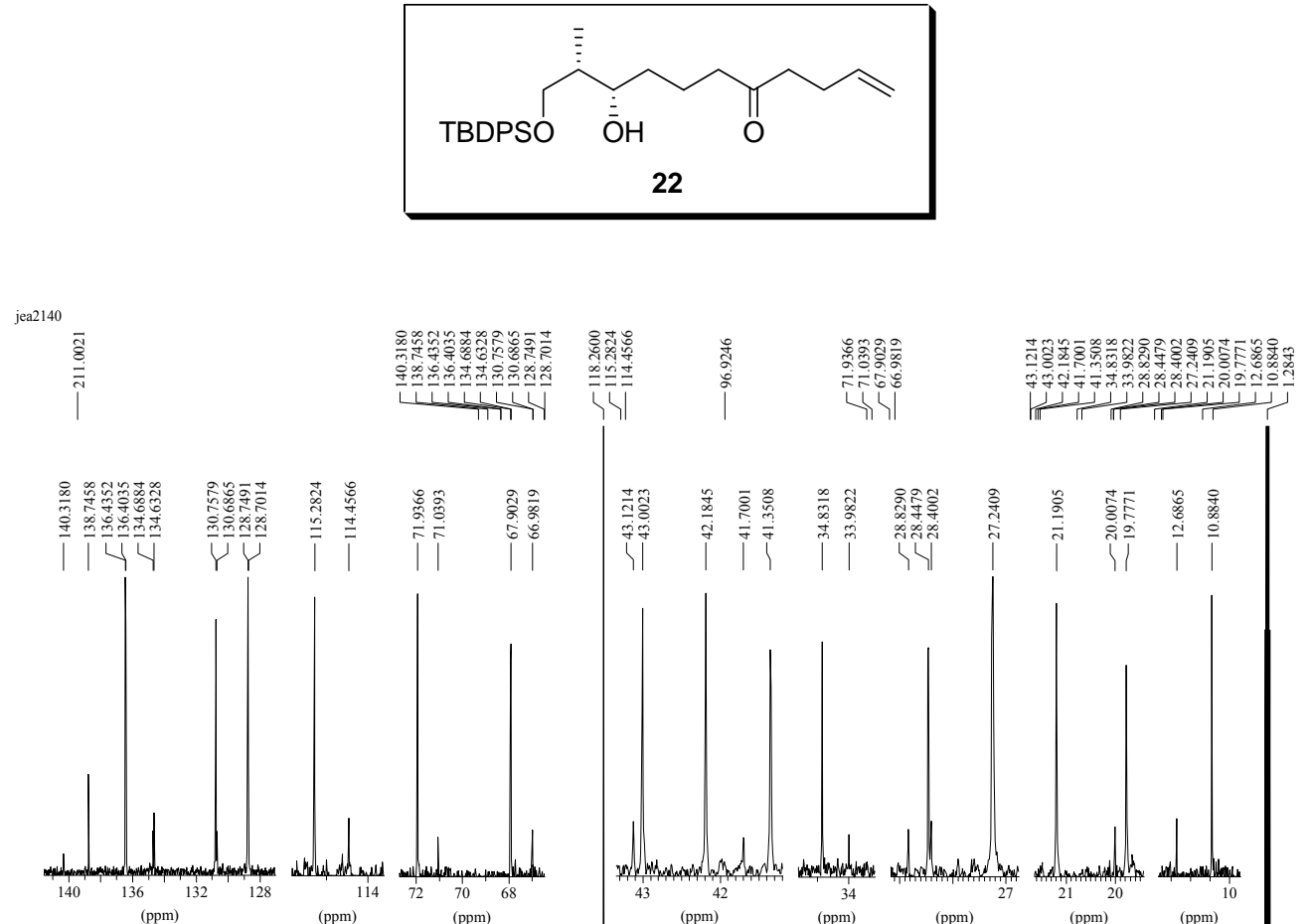

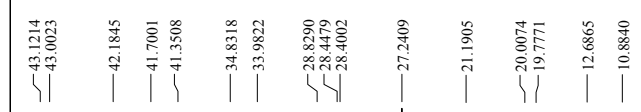

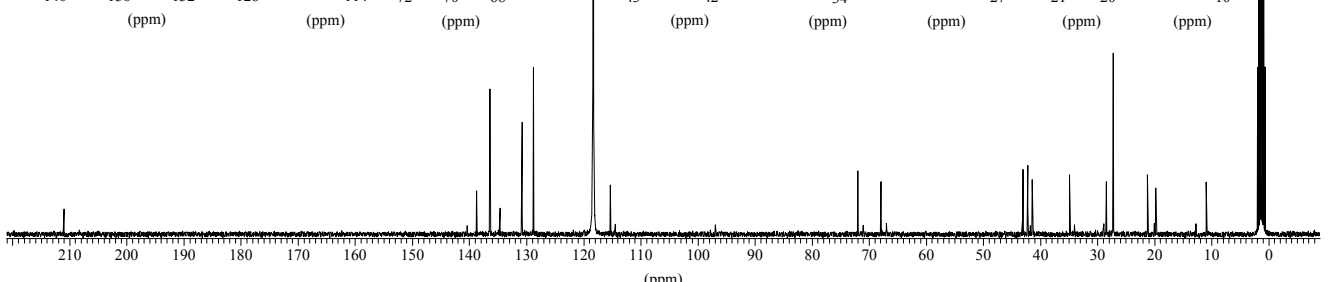




\section{Ketal 23}

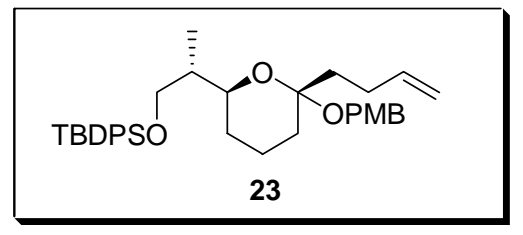

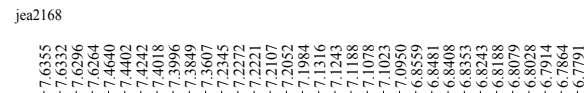

等

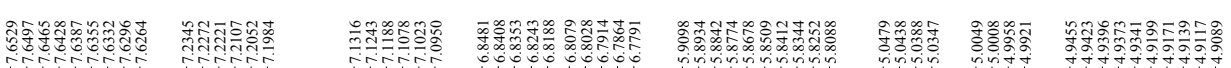
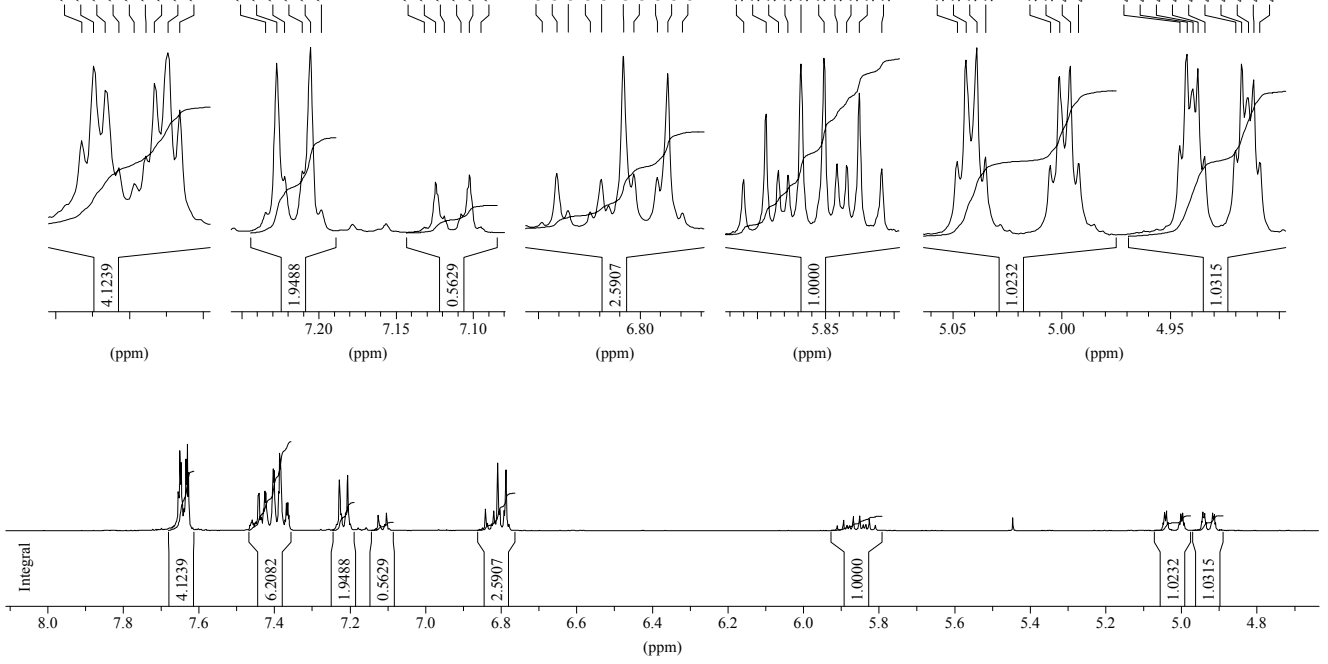

jea2168

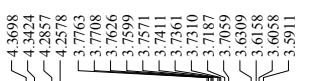

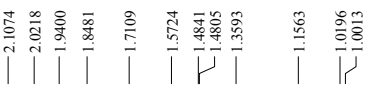

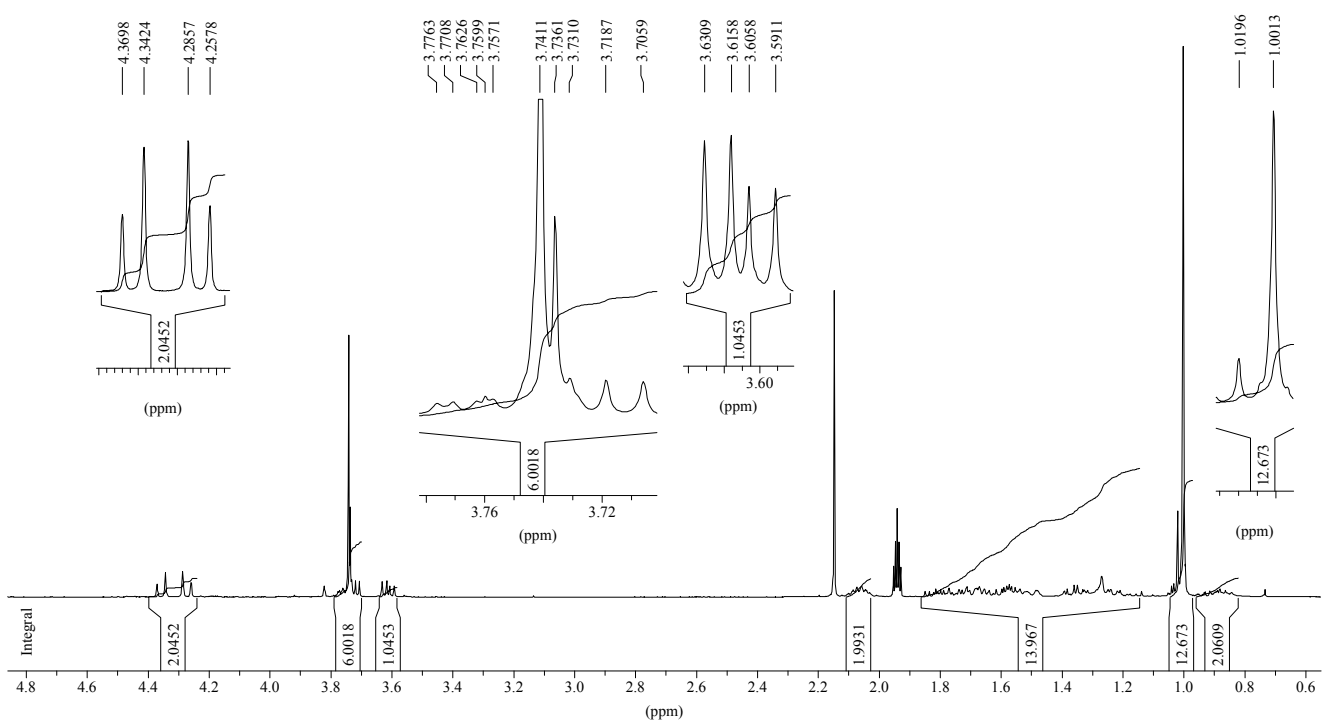


Ketal 23
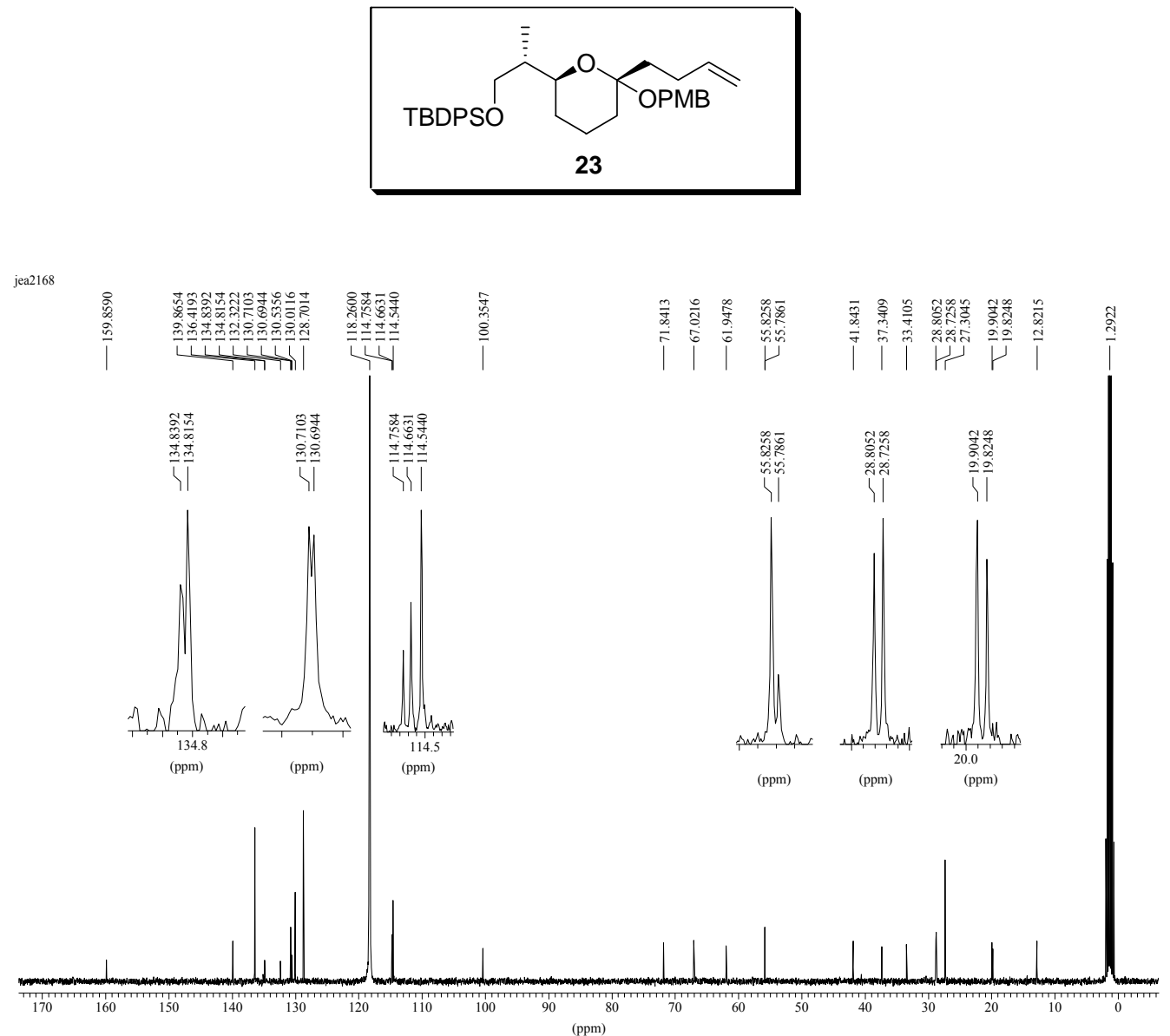


\section{Dihydroxyketal 24}

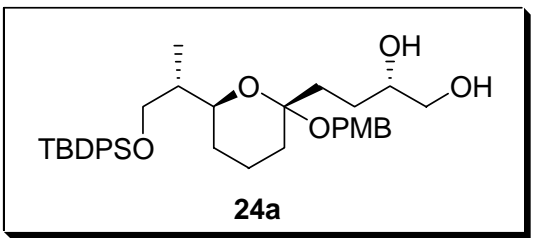

jea2171

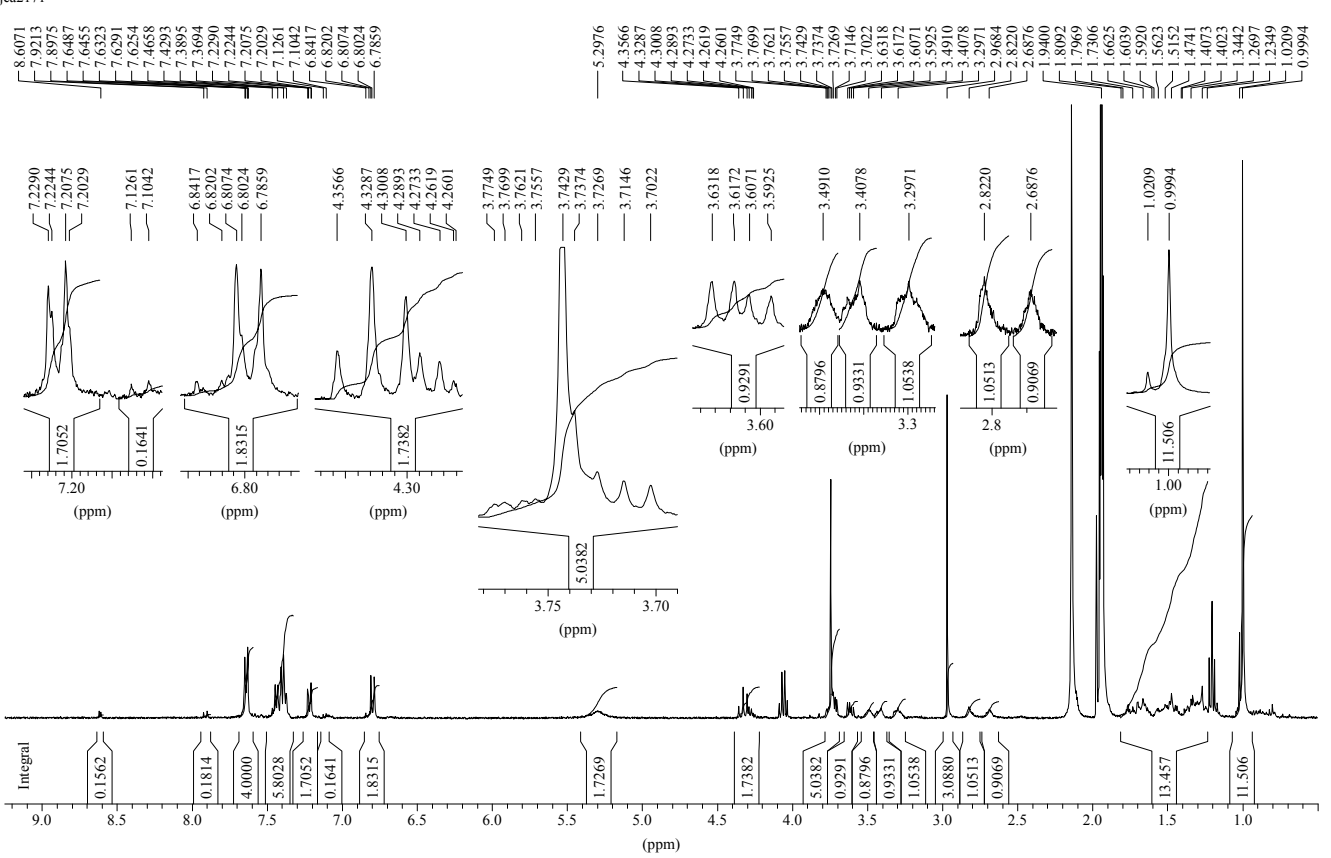

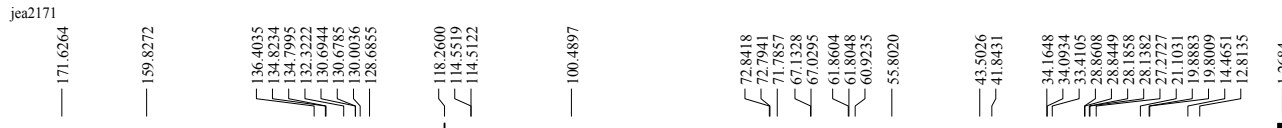

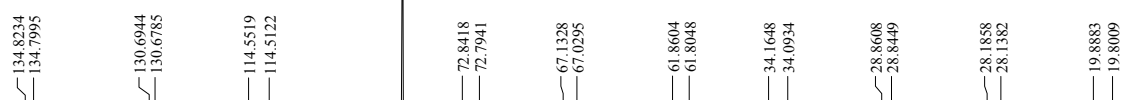
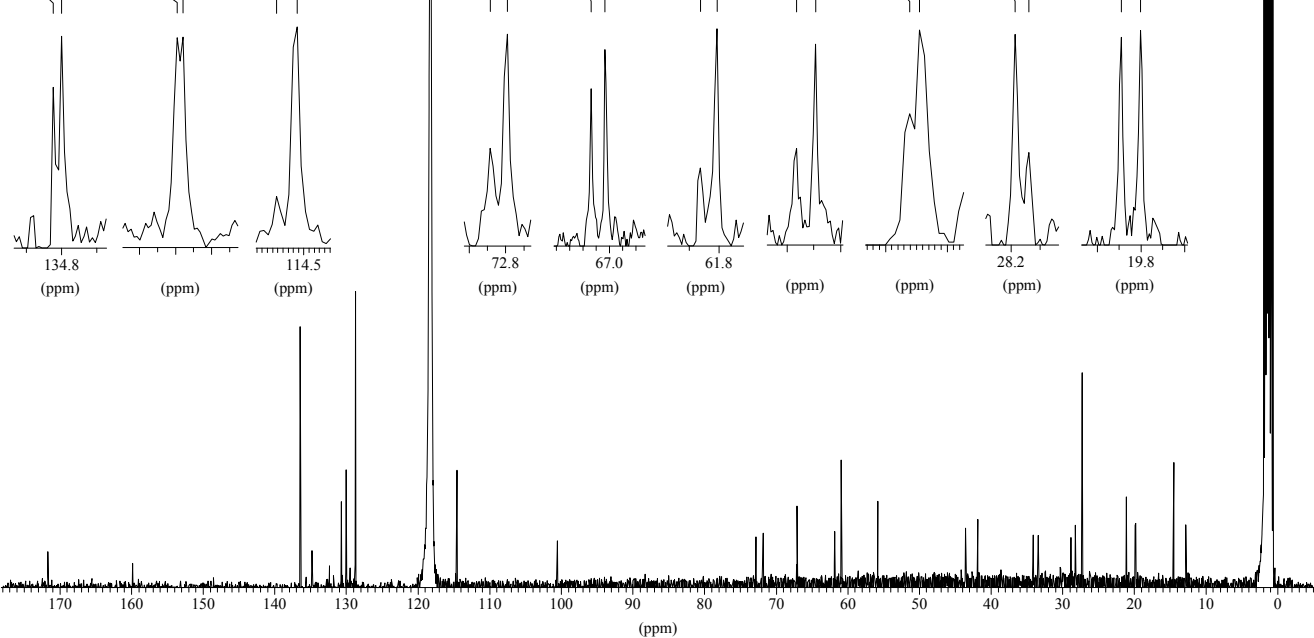
Pivalate 25
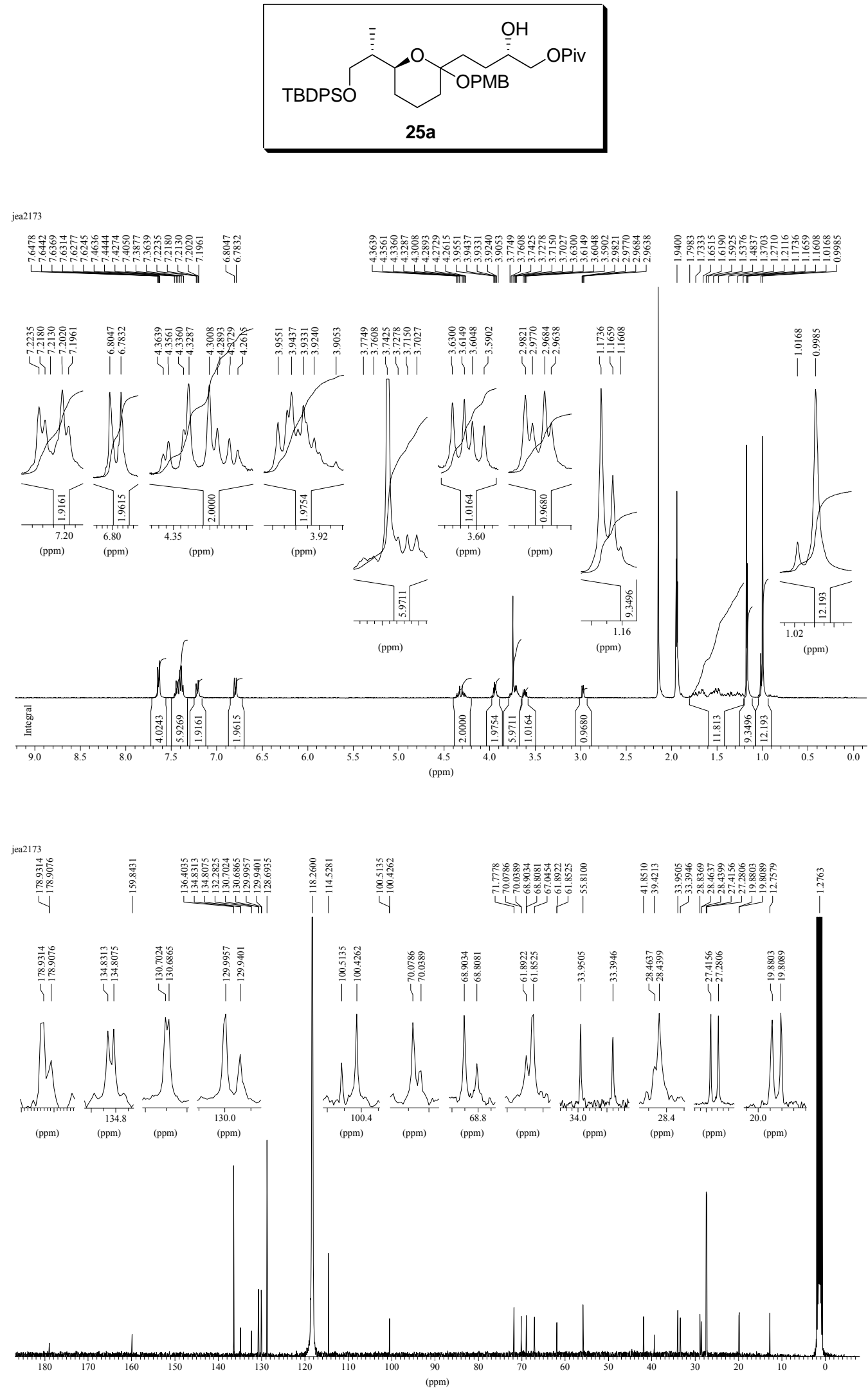


\section{Spiroketal 12}
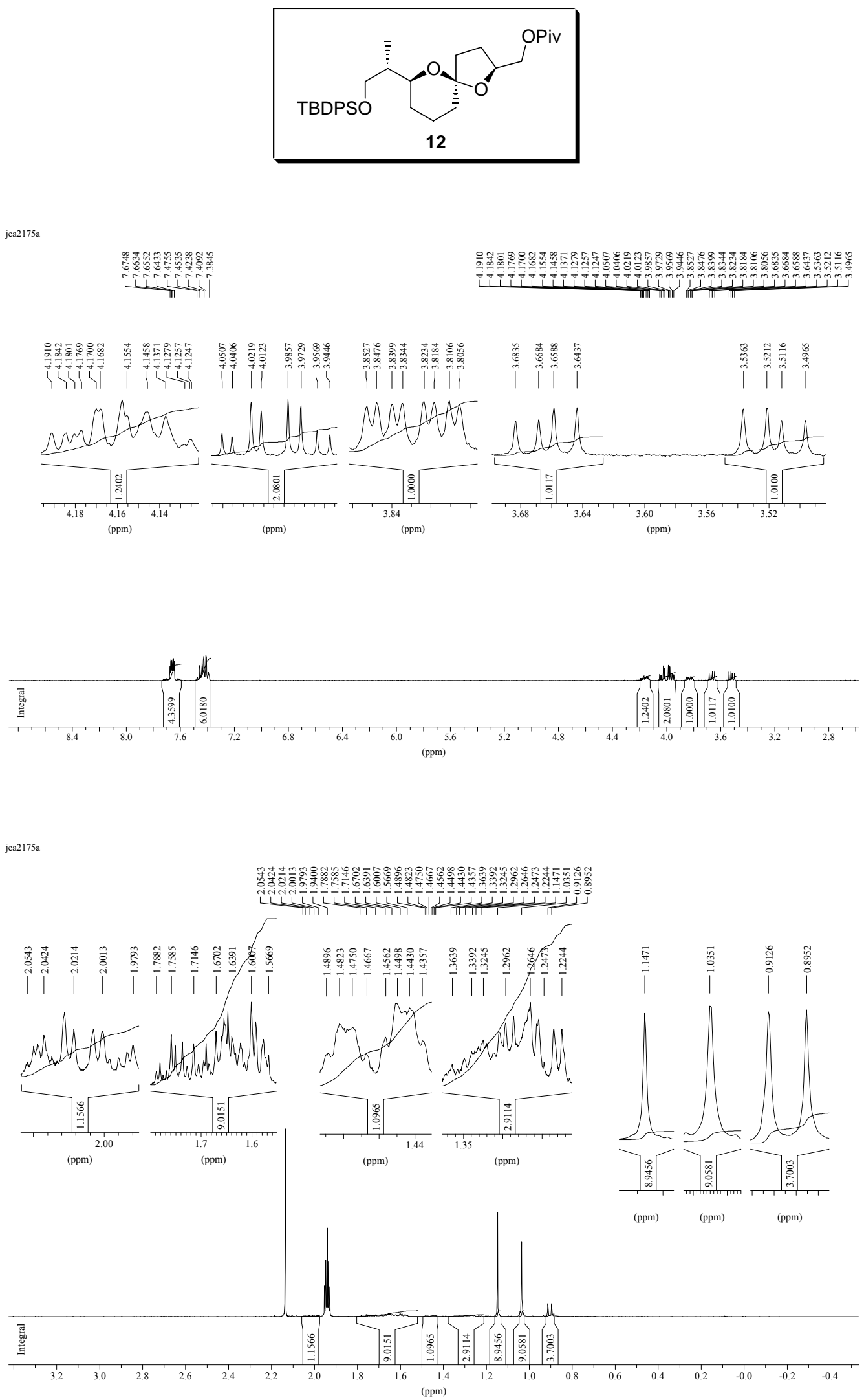
Spiroketal 12
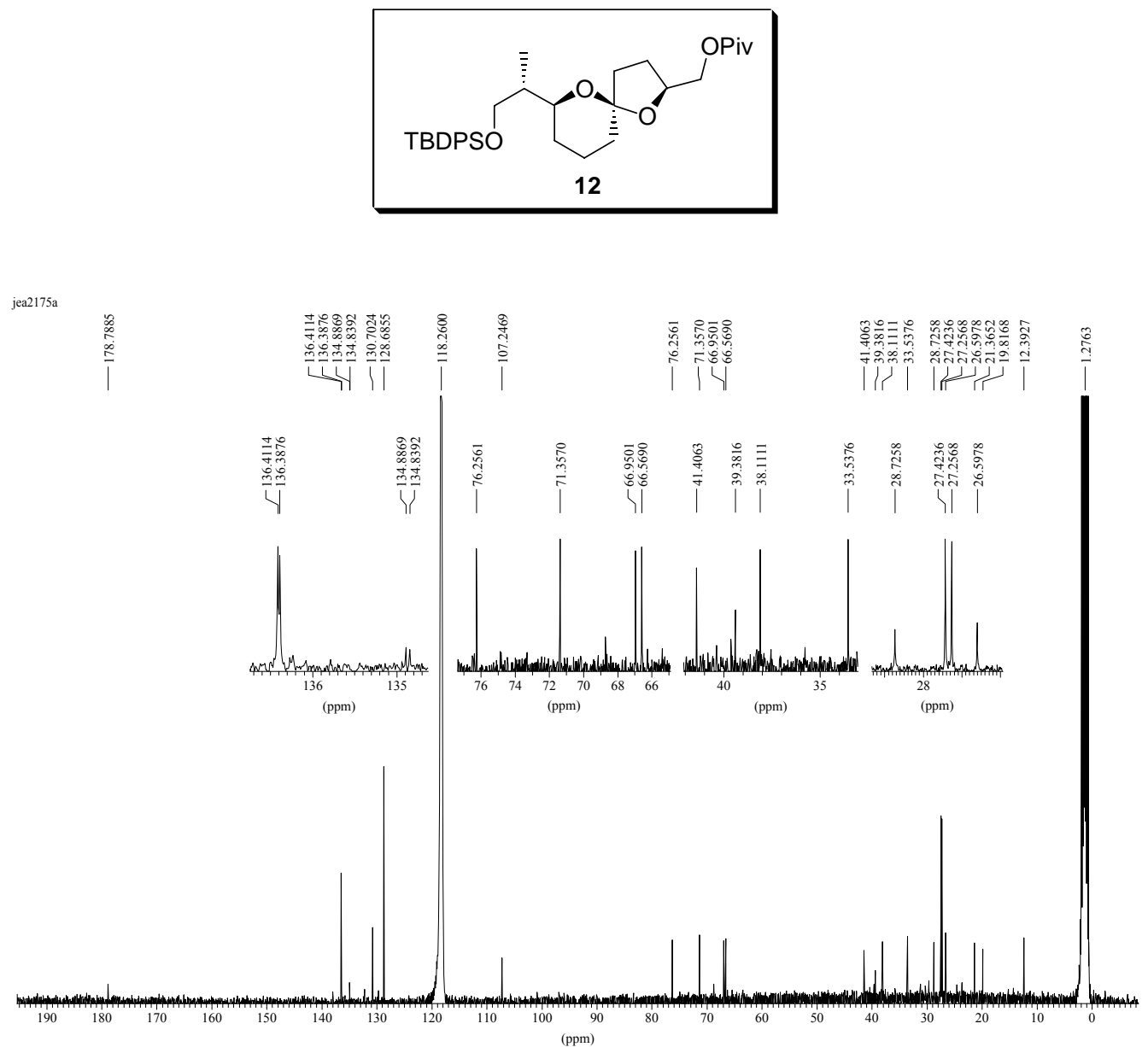


\section{Spiroketal 26}

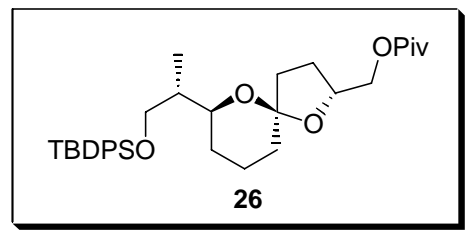

jea2175b

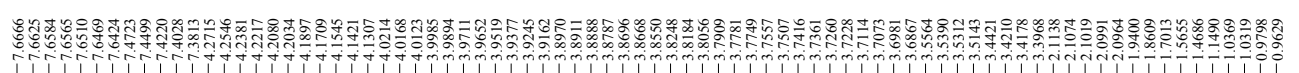

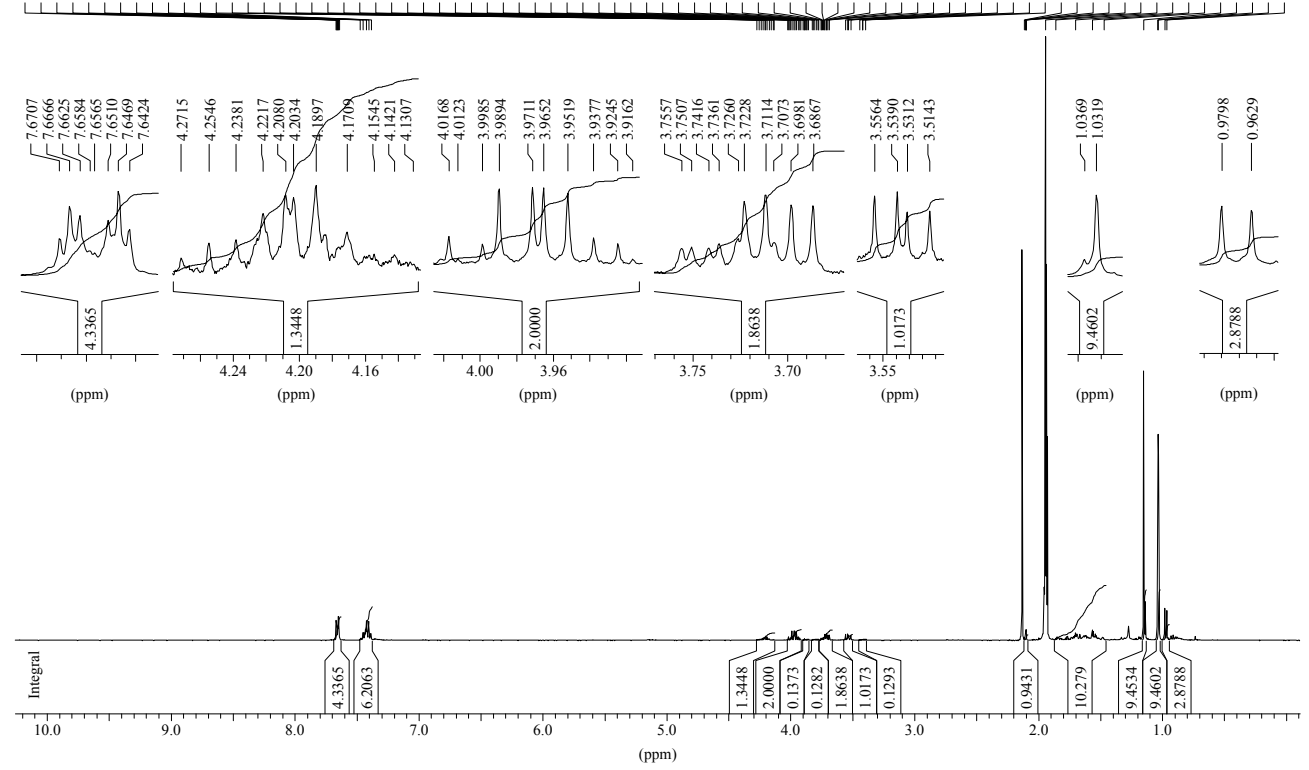

jea2175b

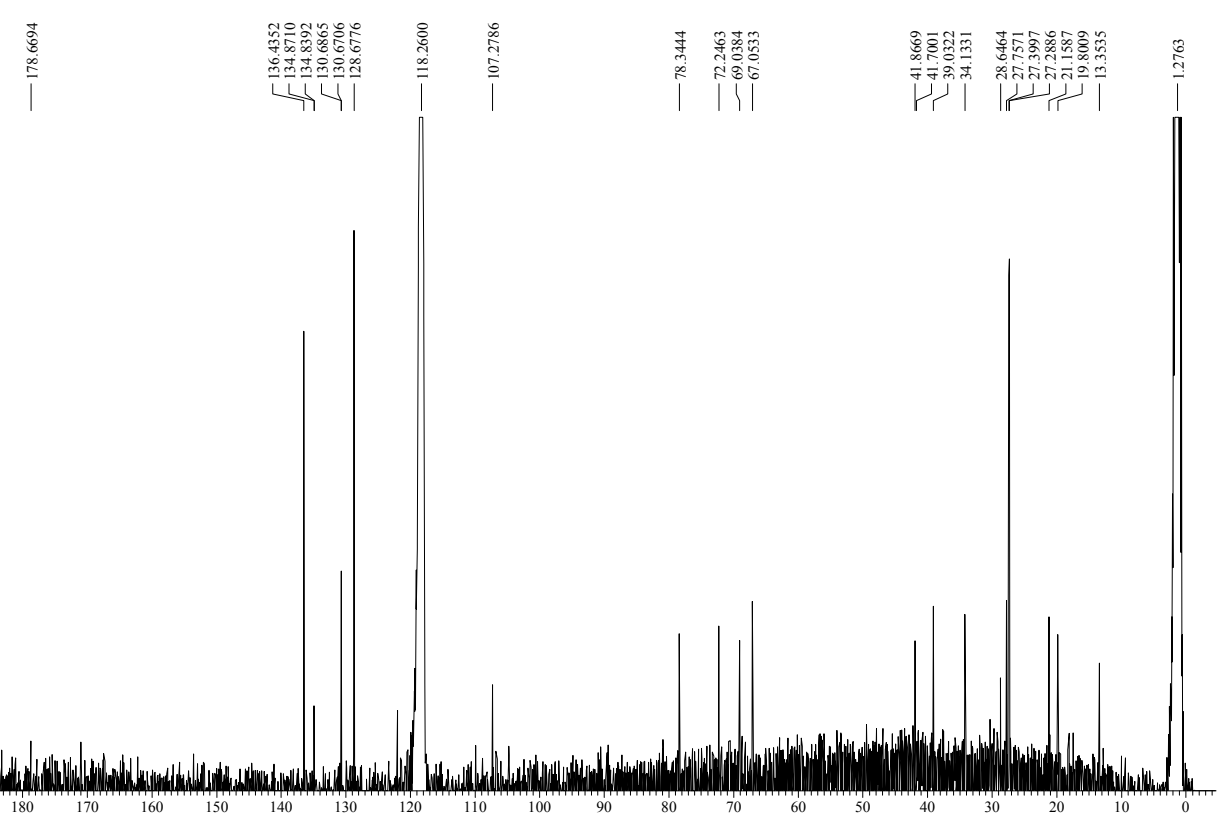

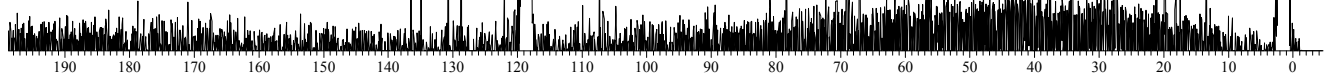




\section{Spiroketal 11}

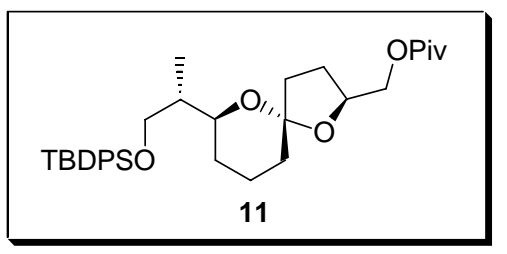

jea2225

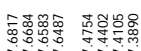

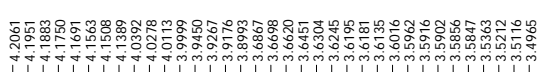

|
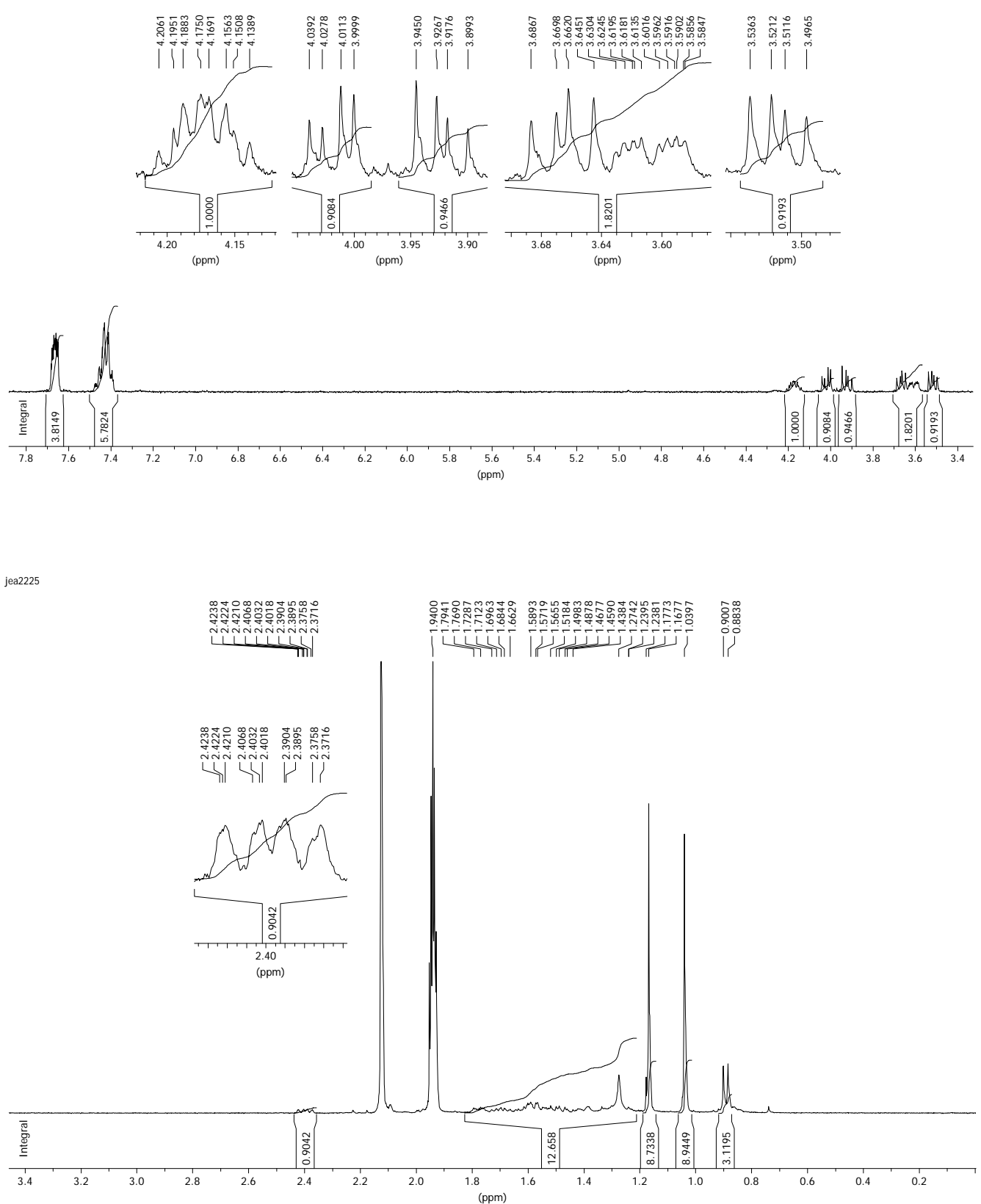
Spiroketal 11
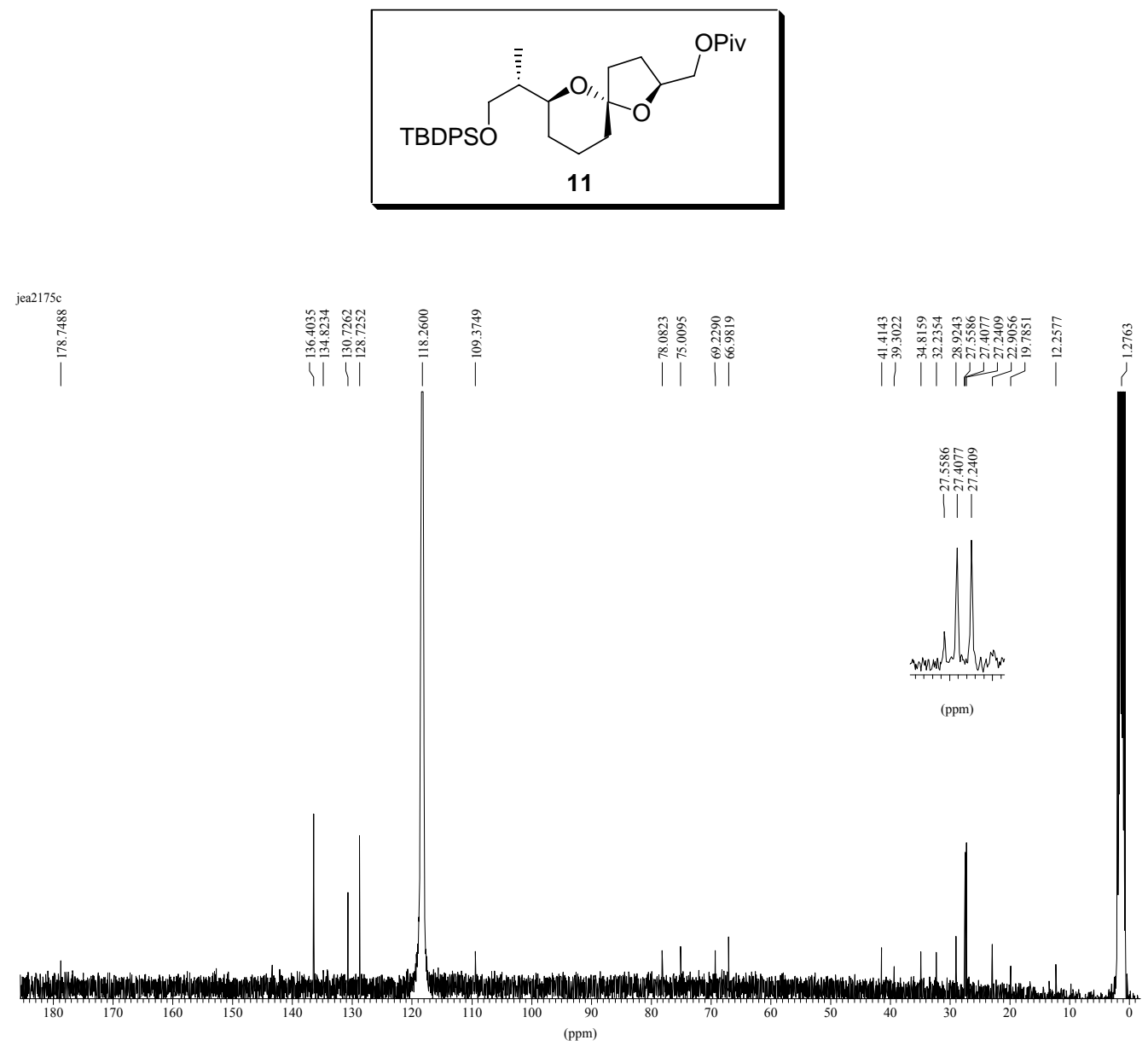